\title{
ON THE KLAINERMAN-MACHEDON CONJECTURE FOR THE QUANTUM BBGKY HIERARCHY WITH SELF-INTERACTION
}

\author{
XUWEN CHEN AND JUSTIN HOLMER
}

\begin{abstract}
We consider the 3D quantum BBGKY hierarchy which corresponds to the $N$-particle Schrödinger equation. We assume the pair interaction is $N^{3 \beta-1} V\left(N^{\beta} \bullet\right.$ ). For interaction parameter $\beta \in\left(0, \frac{2}{3}\right)$, we prove that, provided an energy bound holds for solutions to the BBKGY hierarchy, the $N \rightarrow \infty$ limit points satisfy the space-time bound conjectured by S. Klainerman and M. Machedon [38] in 2008. The energy bound is proven to hold for $\beta \in\left(0, \frac{3}{5}\right)$ in 23 . This allows, in the case $\beta \in\left(0, \frac{3}{5}\right)$, for the application of the KlainermanMachedon uniqueness theorem and hence implies that the $N \rightarrow \infty$ limit of BBGKY is uniquely determined as a tensor product of solutions to the Gross-Pitaevskii equation when the $N$-body initial data is factorized. The first result in this direction in $3 \mathrm{D}$ was obtained by T. Chen and N. Pavlović [11] for $\beta \in\left(0, \frac{1}{4}\right)$ and subsequently by X. Chen [15] for $\beta \in\left(0, \frac{2}{7}\right.$ ]. We build upon the approach of X. Chen but apply frequency localized Klainerman-Machedon collapsing estimates and the endpoint Strichartz estimate in the estimate of the "potential part" to extend the range to $\beta \in\left(0, \frac{2}{3}\right)$. Overall, this provides an alternative approach to the mean-field program by L. Erdös, B. Schlein, and H.-T. Yau [23], whose uniqueness proof is based upon Feynman diagram combinatorics.
\end{abstract}

\section{Contents}

1. Introduction

1.1. Organization of the paper

1.2. Acknowledgements

2. Proof of the main theorem

3. Estimate of the potential part

3.1. A simpler proof in the case $\beta \in\left(0, \frac{2}{5}\right) \quad 12$

3.2. The case $\beta \in\left(0, \frac{2}{3}\right)$

4. Collapsing and Strichartz estimates 21

4.1. Various forms of collapsing estimates 22

4.2. A Strichartz estimate 29

Appendix A. The topology on the density matrices

Appendix B. Proof of estimates (2.8) and (2.10)

References

Date: V2 for JEMS, 02/04/2014.

2010 Mathematics Subject Classification. Primary 35Q55, 35A02, 81V70; Secondary 35A23, 35B45.

Key words and phrases. BBGKY Hierarchy, $N$-particle Schrödinger Equation, Klainerman-Machedon Space-time Bound, Quantum Kac's Program. 


\section{INTRODUCTION}

The 3D quantum BBGKY (Bogoliubov-Born-Green-Kirkwood-Yvon) hierarchy is generated from the $N$-body Hamiltonian evolution $\psi_{N}(t)=e^{i t H_{N}} \psi_{N, 0}$ with symmetric initial datum and the $N$-body Hamiltonian is given by

$$
H_{N}=-\triangle_{\mathbf{x}_{N}}+\frac{1}{N} \sum_{1 \leqslant i<j \leqslant N} N^{3 \beta} V\left(N^{\beta}\left(x_{i}-x_{j}\right)\right) .
$$

In the above, $t \in \mathbb{R}, \mathbf{x}_{N}=\left(x_{1}, x_{2}, \cdots, x_{N}\right) \in \mathbb{R}^{3 N}, \triangle_{\mathbf{x}_{N}}$ denotes the standard Laplacian with respect to the variables $\mathbf{x}_{N} \in \mathbb{R}^{3 N}$, the factor $1 / N$ in (1.1) is to make sure that the interactions are proportional to the number of particles, and the pair interaction $N^{3 \beta} V\left(N^{\beta}\left(x_{i}-x_{j}\right)\right)$ is an approximation to the Dirac $\delta$ function which matches the Gross-Pitaevskii description of Bose-Einstein condensation that the many-body effect should be modeled by a strong onsite self-interaction. Since $\psi_{N} \overline{\psi_{N}}$ is a probability density, we define the marginal densities $\left\{\gamma_{N}^{(k)}\left(t, \mathbf{x}_{k} ; \mathbf{x}_{k}^{\prime}\right)\right\}_{k=1}^{N}$ by

$$
\gamma_{N}^{(k)}\left(t, \mathbf{x}_{k} ; \mathbf{x}_{k}^{\prime}\right)=\int \psi_{N}\left(t, \mathbf{x}_{k}, \mathbf{x}_{N-k}\right) \overline{\psi_{N}}\left(t, \mathbf{x}_{k}^{\prime}, \mathbf{x}_{N-k}\right) d \mathbf{x}_{N-k}, \mathbf{x}_{k}, \mathbf{x}_{k}^{\prime} \in \mathbb{R}^{3 k} .
$$

Then we have that $\left\{\gamma_{N}^{(k)}\left(t, \mathbf{x}_{k} ; \mathbf{x}_{k}^{\prime}\right)\right\}_{k=1}^{N}$ is a sequence of trace class operator kernels which are symmetric, in the sense that

$$
\gamma_{N}^{(k)}\left(t, \mathbf{x}_{k}, \mathbf{x}_{k}^{\prime}\right)=\overline{\gamma_{N}^{(k)}\left(t, \mathbf{x}_{k}^{\prime}, \mathbf{x}_{k}\right)}
$$

and

$$
\gamma_{N}^{(k)}\left(t, x_{\sigma(1)}, \cdots x_{\sigma(k)}, x_{\sigma(1)}^{\prime}, \cdots x_{\sigma(k)}^{\prime}\right)=\gamma_{N}^{(k)}\left(t, x_{1}, \cdots, x_{k}, x_{1}^{\prime}, \cdots, x_{k}^{\prime}\right),
$$

for any permutation $\sigma$, and satisfy the 3D quantum BBGKY hierarchy of equations which written in operator form is

$$
\begin{aligned}
i \partial_{t} \gamma_{N}^{(k)}+\left[\triangle_{\mathbf{x}_{k}}, \gamma_{N}^{(k)}\right]= & \frac{1}{N} \sum_{1 \leqslant i<j \leqslant k}\left[V_{N}\left(x_{i}-x_{j}\right), \gamma_{N}^{(k)}\right] \\
& +\frac{N-k}{N} \sum_{j=1}^{k} \operatorname{Tr}_{k+1}\left[V_{N}\left(x_{j}-x_{k+1}\right), \gamma_{N}^{(k+1)}\right]
\end{aligned}
$$

if we do not distinguish $\gamma_{N}^{(k)}$ as a kernel and the operator it defines 1 Here the operator $V_{N}(x)$ represents multiplication by the function $V_{N}(x)$, where

$$
V_{N}(x)=N^{3 \beta} V\left(N^{\beta} x\right),
$$

and $\operatorname{Tr}_{k+1}$ means taking the $k+1$ trace, for example,

$$
\operatorname{Tr}_{k+1} V_{N}\left(x_{j}-x_{k+1}\right) \gamma_{N}^{(k+1)}=\int V_{N}\left(x_{j}-x_{k+1}\right) \gamma_{N}^{(k+1)}\left(t, \mathbf{x}_{k}, x_{k+1} ; \mathbf{x}_{k}^{\prime}, x_{k+1}\right) d x_{k+1}
$$

In 2008, S. Klainerman and M. Machedon implicitly made the following conjecture on the solution of the BBGKY hierarchy.

\footnotetext{
${ }^{1}$ From here on out, we consider only the $\beta>0$ case. For $\beta=0$, see $[21,26$, 39, 41, 43, 31, 32, 13, 6] .
} 
Conjecture 1 (Klainerman-Machedon [38]). Assume the interaction parameter $\beta \in(0,1]$. Suppose that the sequence $\left\{\gamma_{N}^{(k)}\left(t, \mathbf{x}_{k} ; \mathbf{x}_{k}^{\prime}\right)\right\}_{k=1}^{N}$ is a solution to the $3 D$ quantum BBGKY hierarchy (1.3) subject to the energy condition: there is a $C_{0}$ (independent of $N$ and $k$ ) such that for any $k \geqslant 0$, there is a $N_{0}(k)$ such that

$$
\forall N \geqslant N_{0}(k), \quad \sup _{t \in \mathbb{R}} \operatorname{Tr}\left(\prod_{j=1}^{k}\left(1-\triangle_{x_{j}}\right)\right) \gamma_{N}^{(k)} \leqslant C_{0}^{k} .
$$

Then, for every finite time $T$, every limit point $\Gamma=\left\{\gamma^{(k)}\right\}_{k=1}^{\infty}$ of $\left\{\Gamma_{N}\right\}_{N=1}^{\infty}=\left\{\left\{\gamma_{N}^{(k)}\right\}_{k=1}^{N}\right\}_{N=1}^{\infty}$ in $\bigoplus_{k \geqslant 1} C\left([0, T], \mathcal{L}_{k}^{1}\right)$ with respect to the product topology $\tau_{\text {prod }}$ (defined in Appendix A]) satisfies the space-time bound: there is a $C$ independent of $j, k$ such that

$$
\int_{0}^{T}\left\|R^{(k)} B_{j, k+1} \gamma^{(k+1)}(t)\right\|_{L_{\mathbf{x}, \mathbf{x}^{\prime}}^{2}} d t \leqslant C^{k}
$$

where $\mathcal{L}_{k}^{1}$ is the space of trace class operators on $L^{2}\left(\mathbb{R}^{3 k}\right), R^{(k)}=\prod_{j=1}^{k}\left(\left|\nabla_{x_{j}}\right|\left|\nabla_{x_{j}^{\prime}}\right|\right)$, and

$$
B_{j, k+1}=\operatorname{Tr}_{k+1}\left[\delta\left(x_{j}-x_{k+1}\right), \gamma^{(k+1)}\right] .
$$

Though Conjecture 1 was not stated explicitly in [38], as we will explain after stating Theorem 1.1, the bound (1.6) is necessary to implement Klainerman-Machedon's powerful and flexible approach in the most involved part of the quantum Kac's program which mathematically proves the cubic nonlinear Schrödinger equation (NLS) as the $N \rightarrow \infty$ limit of quantum $N$-body dynamics. Kirkpatrick-Schlein-Staffilani [36] completely solved the $\mathbb{T}^{2}$ version of Conjecture 1 and were the first to successfully implement such an approach. However, Conjecture 1, the $\mathbb{R}^{3}$ version as stated, was fully open until recently. T. Chen and Pavlović [11] have been able to prove Conjecture 1 for $\beta \in(0,1 / 4)$. In [15], X.C simplified and extended the result to the range of $\beta \in(0,2 / 7]$. We devote this paper to proving Conjecture 1 for $\beta \in(0,2 / 3)$. In particular, we surpass the self-interaction thresold2, namely $\beta=1 / 3$. To be specific, we prove the following theorem.

Theorem 1.1 (Main theorem). Assume the interaction parameter $\beta \in(0,2 / 3)$ and the pair interaction $V \in L^{1} \cap W^{2, \frac{6}{5}+}$. Under condition (2.5), every limit point $\Gamma=\left\{\gamma^{(k)}\right\}_{k=1}^{\infty}$ of $\left\{\Gamma_{N}\right\}_{N=1}^{\infty}$ satisfies the Klainerman-Machedon space-time bound (1.6).

Establishing the $N \rightarrow \infty$ limit of hierarchy (1.3) justifies the mean-field limit in the GrossPitaevskii theory. Such an approach was first proposed by Spohn [44] and can be regarded as a quantum version of Kac's program. We see that, as $N \rightarrow \infty$, hierarchy (1.3) formally converges to the infinite Gross-Pitaevskii hierarchy

$$
i \partial_{t} \gamma^{(k)}+\left[\triangle_{\mathbf{x}_{k}}, \gamma^{(k)}\right]=\left(\int V(x) d x\right) \sum_{j=1}^{k} \operatorname{Tr}_{k+1}\left[\delta\left(x_{j}-x_{k+1}\right), \gamma^{(k+1)}\right] .
$$

\footnotetext{
${ }^{2}$ We will explain why we call the $\beta>1 / 3$ case self-interaction later in this introduction.
} 
When the initial data is factorized

$$
\gamma^{(k)}\left(0, \mathbf{x}_{k} ; \mathbf{x}_{k}^{\prime}\right)=\prod_{j=1}^{k} \phi_{0}\left(x_{j}\right) \bar{\phi}_{0}\left(x_{j}\right)
$$

hierarchy (1.7) has a special solution

$$
\gamma^{(k)}\left(t, \mathbf{x}_{k} ; \mathbf{x}_{k}^{\prime}\right)=\prod_{j=1}^{k} \phi\left(t, x_{j}\right) \bar{\phi}\left(t, x_{j}\right),
$$

if $\phi$ solves the cubic NLS

$$
i \partial_{t} \phi=-\triangle_{x} \phi+\left(\int V(x) d x\right)|\phi|^{2} \phi .
$$

Thus such a limit process shows that, in an appropriate sense,

$$
\lim _{N \rightarrow \infty} \gamma_{N}^{(k)}=\prod_{j=1}^{k} \phi\left(t, x_{j}\right) \bar{\phi}\left(t, x_{j}\right),
$$

hence justifies the mean-field limit.

Such a limit in 3D was first proved in a series of important papers [20, 22, 23, 24, 25] by Elgart, Erdös, Schlein, and Yau 3 Briefly, the Elgart-Erdös-Schlein-Yau approach described as the following:

Step A. Prove that, with respect to the topology $\tau_{\text {prod }}$ defined in Appendix $\mathrm{A}$, the sequence $\left\{\Gamma_{N}\right\}_{N=1}^{\infty}$ is compact in the space $\left.\bigoplus_{k \geqslant 1} C\left([0, T], \mathcal{L}^{1}\left(\mathbb{R}^{3 k}\right)\right)\right)$.

Step B. Prove that every limit point $\Gamma=\left\{\gamma^{(k)}\right\}_{k=1}^{\infty}$ of $\left\{\Gamma_{N}\right\}_{N=1}^{\infty}$ must verify hierarchy (1.7).

Step $C$. Prove that, in the space in which the limit points from Step B lie, there is a unique solution to hierarchy (1.7). Thus $\left\{\Gamma_{N}\right\}_{N=1}^{\infty}$ is a compact sequence with only one limit point. Hence $\Gamma_{N} \rightarrow \Gamma$ as $N \rightarrow \infty$.

In 2007, Erdös, Schlein, and Yau obtained the first uniqueness theorem of solutions [23, Theorem 9.1] to the hierarchy (1.7). The proof is surprisingly delicate - it spans 63 pages and uses complicated Feynman diagram techniques. The main difficulty is that hierarchy (1.7) is a system of infinitely coupled equations. Briefly, [23, Theorem 9.1] is the following:

Theorem 1.2 (Erdös-Schlein-Yau uniqueness [23, Theorem 9.1]). There is at most one nonnegative symmetric operator sequence $\left\{\gamma^{(k)}\right\}_{k=1}^{\infty}$ that solves hierarchy (1.7) subject to the energy condition

$$
\sup _{t \in[0, T]} \operatorname{Tr}\left(\prod_{j=1}^{k}\left(1-\triangle_{x_{j}}\right)\right) \gamma^{(k)} \leqslant C^{k}
$$

\footnotetext{
${ }^{3}$ Around the same time, there was the $1 \mathrm{D}$ work [1].

${ }^{4}$ See [5, 30, 42] for different approaches.
} 
In [38], based on their null form paper [37], Klainerman and Machedon gave a different proof of the uniqueness of hierarchy (1.7) in a space different from that used in [23, Theorem 9.1]. The proof is shorter (13 pages) than the proof of [23, Theorem 9.1]. Briefly, [38, Theorem 1.1] is the following:

Theorem 1.3 (Klainerman-Machedon uniqueness [38, Theorem 1.1]). There is at most one symmetric operator sequence $\left\{\gamma^{(k)}\right\}_{k=1}^{\infty}$ that solves hierarchy (1.7) subject to the space-time bound (1.6).

For special cases like (1.8), condition (1.10) is actually

$$
\sup _{t \in[0, T]}\left\|\left\langle\nabla_{x}\right\rangle \phi\right\|_{L^{2}} \leqslant C
$$

while condition (1.6) means

$$
\int_{0}^{T}\left\|\left|\nabla_{x}\right|\left(|\phi|^{2} \phi\right)\right\|_{L^{2}} d t \leqslant C .
$$

When $\phi$ satisfies NLS (1.9), both are known. In fact, due to the Strichartz estimate [34], (1.11) implies (1.12), that is, condition (1.6) seems to be a bit weaker than condition (1.10). The proof of [38, Theorem 1.1] (13 pages) is also considerably shorter than the proof of [23, Theorem 9.1] (63 pages). It is then natural to wonder whether [38, Theorem 1.1] simplifies Step C. To answer such a question it is necessary to know whether the limit points in Step B satisfy condition (1.6), that is, whether Conjecture 1 holds.

Away from curiosity, there are realistic reasons to study Conjecture11. While [23, Theorem 9.1] is a powerful theorem, it is very difficult to adapt such an argument to various other interesting and colorful settings: a different spatial dimension, a three-body interaction instead of a pair interaction, or the Hermite operator instead of the Laplacian. The last situation mentioned is physically important. On the one hand, all the known experiments of BEC use harmonic trapping to stabilize the condensate [2, 18, 7, 35, 45]. On the other hand, different trapping strength produces quantum behaviors which do not exist in the Boltzmann limit of classical particles nor in the quantum case when the trapping is missing and have been experimentally observed [27, 46, 17, 33, 19]. The Klainerman-Machedon approach applies easily in these meaningful situations ([36, 9, 14, 15, 16, 28]). Thus proving Conjecture 1 actually helps to advance the study of quantum many-body dynamic and the mean-field approximation in the sense that it provides a flexible and powerful tool in 3D.

The well-posedness theory of the Gross-Pitaevskii hierarchy (1.7) subject to general initial datum also requires that the limits of the BBGKY hierarchy (1.3) lie in the space in which the space-time bound (1.6) holds. See [8, 10, 11].

As pointed out in [20], the study of the Hamiltonian (1.1) is of particular interest when $\beta \in(1 / 3,1]$. The reason is the following. In physics, the initial datum $\psi_{N}(0)$ of the Hamiltonian evolution $e^{i t H_{N}} \psi_{N}(0)$ is usually assumed to be close to the ground state of the Hamiltonian

$$
H_{N, 0}=-\triangle_{\mathbf{x}_{N}}+\omega_{0}^{2}\left|\mathbf{x}_{N}\right|^{2}+\frac{1}{N} \sum_{1 \leqslant i<j \leqslant N} N^{3 \beta} V\left(N^{\beta}\left(x_{i}-x_{j}\right)\right)
$$


The preparation of the available experiments and the mathematical work [40] by Lieb, Seiringer, Solovej and Yngvason confirm this assumption. Such an initial datum $\psi_{N}(0)$ is localized in space. We can assume all $N$ particles are in a box of length 1 . Let the effective radius of the pair interaction $V$ be $a$, then the effective radius of $V_{N}$ is about $a / N^{\beta}$. Thus every particle in the box interacts with $\left(a / N^{\beta}\right)^{3} \times N$ other particles. Thus, for $\beta>1 / 3$ and large $N$, every particle interacts with only itself. This exactly matches the Gross-Pitaevskii theory that the many-body effect should be modeled by a strong on-site self-interaction. Therefore, for the mathematical justification of the Gross-Pitaevskii theory, it is of particular interest to prove Conjecture 1 for self-interaction $(\beta>1 / 3)$ as well.

To the best of our knowledge, the main theorem (Theorem 1.1) in the current paper is the first result in proving Conjecture 1 for self-interaction $(\beta>1 / 3)$. For $\beta \leqslant 1 / 3$, the first progress of Conjecture 1 is the $\beta \in(0,1 / 4)$ work [11] by T. Chen and N. Pavlović and then the $\beta \in(0,2 / 7]$ work [15] by X.C. As a matter of fact, the main theorem (Theorem 1.1) in the current paper has already fulfilled the original intent of [38], namely, simplifying the uniqueness argument of [23], because [23] deals with $\beta \in(0,3 / 5)$. Conjecture 1 for $\beta \in[2 / 3,1]$ is still open.

1.1. Organization of the paper. In $\oint 2$, we outline the proof of Theorem 1.1. The overall pattern follows that introduced by X.C. [15], who obtained Theorem 1.1 for $\beta \in\left(0, \frac{2}{7}\right]$. Let $P_{\leq M}^{(k)}$ be the Littlewood-Paley projection defined in (2.1). Theorem 1.1 will follow once it is established that for all $M \geq 1$, there exists $N_{0}$ depending on $M$ such that for all $N \geq N_{0}$, there holds

$$
\left\|P_{\leq M}^{(k)} R^{(k)} B_{N, j, k+1} \gamma_{N}^{(k+1)}(t)\right\|_{L_{T}^{1} L_{\mathbf{x}, \mathbf{x}^{\prime}}^{2}} \leqslant C^{k}
$$

where $B_{N, j, k+1}$ is defined by (2.3). Substituting the Duhamel-Born expansion, carried out to coupling level $l_{c}$, of the BBGKY hierarchy, this is reduced to proving analogous bounds on the free part, potential part, and interaction part, defined in \$2. Each part is reduced via the Klainerman-Machedon board game. Estimates for the free part and interaction part were previously obtained by X.C. [15] but are reproduced here for convenience in Appendix $\mathrm{B}$. For the estimate of the interaction part, one takes $l_{c}=\ln N$, the utility of which was first observed by T. Chen and N. Pavlović [11].

The main new achievement of our paper is the improved estimates on the potential part, which are discussed in \$3 . We make use of the endpoint Strichartz estimate, phrased in terms of the $X_{b}$ norm, in place of the Sobolev inequality employed by X.C [15]. We also introduce frequency localized versions of the Klainerman-Machedon collapsing estimates, allowing us to exploit the frequency localization in (1.13). Specifically, the operator $P_{\leq M}^{(k)}$ does not commute with $B_{N, j, k+1}$, however, the composition $P_{\leq M_{k}}^{(k)} B_{N, j, k+1} P_{\sim M_{k+1}}^{(k+1)}$ enjoys better bounds if $M_{k+1} \gg M_{k}$. We prove the Strichartz estimate and the frequency localized KlainermanMachedon collapsing estimates in 4 . Frequency localized space-time techniques of this type were introduced by Bourgain [4, Chapter IV, §3] into the study of the well-posedness for nonlinear Schrödinger equations and other nonlinear dispersive PDE.

In X.C. [15], (1.13) is obtained without the frequency localization $P_{\leq M}^{(k)}$ for $\beta \in\left(0, \frac{2}{7}\right]$. In Theorem 3.2, we prove that this estimate still holds without frequency localization for 
$\beta \in\left(0, \frac{2}{5}\right)$ by using the Strichartz estimate alone. This already surpasses the self-interaction threshold $\beta=\frac{1}{3}$. For the purpose of proving Conjecture 1, the frequency localized estimate (1.13) is equally good, but allows us to achieve higher $\beta$.

1.2. Acknowledgements. J.H. was supported in part by NSF grant DMS-0901582 and a Sloan Research Fellowship (BR-4919), and X.C. received travel support from the same Sloan Fellowship to visit U. Maryland. We would like to thank T. Chen, M. Grillakis, M. Machedon, and N. Pavlović for very helpful discussions related to this work, and we would like to thank the anonymous referee for many helpful suggestions.

\section{PROOF OF THE MAIN THEOREM}

We establish Theorem 1.1 in this section. For simplicity of notation, we denote $\|\cdot\|_{L^{p}[0, T] L_{\mathbf{x}, \mathbf{x}^{\prime}}^{2}}$ by $\|\cdot\|_{L_{T}^{p} L_{\mathbf{x}, \mathbf{x}^{\prime}}^{2}}$ and denote $\|\cdot\|_{L_{t}^{p}(\mathbb{R}) L_{\mathbf{x}, \mathbf{x}^{\prime}}^{2}}$ by $\|\cdot\|_{L_{t}^{p} L_{\mathbf{x}, \mathbf{x}^{\prime}}^{2}}$. Let us begin by introducing some notation for Littlewood-Paley theory. Let $P_{\leq M}^{i}$ be the projection onto frequencies $\leq M$ and $P_{M}^{i}$ the analogous projections onto frequencies $\sim M$, acting on functions of $x_{i} \in \mathbb{R}^{3}$ (the $i$ th coordinate). We take $M$ to be a dyadic frequency range $2^{\ell} \geq 1$. Similarly, we define $P_{\leq M}^{i^{\prime}}$ and $P_{M}^{i^{\prime}}$, which act on the variable $x_{i}^{\prime}$. Let

$$
P_{\leq M}^{(k)}=\prod_{i=1}^{k} P_{\leq M}^{i} P_{\leq M}^{i^{\prime}} .
$$

To establish Theorem 1.1, it suffices to prove the following theorem.

Theorem 2.1. Under the assumptions of Theorem 1.1, there exists a $C$ (independent of $k, M, N)$ such that for each $M \geq 1$ there exists $N_{0}$ (depending on $M$ ) such that for $N \geqslant N_{0}$, there holds

$$
\left\|P_{\leq M}^{(k)} R^{(k)} B_{N, j, k+1} \gamma_{N}^{(k+1)}(t)\right\|_{L_{T}^{1} L_{\mathbf{x}, \mathbf{x}^{\prime}}^{2}} \leqslant C^{k}
$$

where

$$
B_{N, j, k+1} \gamma_{N}^{(k+1)}=\operatorname{Tr}_{k+1}\left[V_{N}\left(x_{j}-x_{k+1}\right), \gamma_{N}^{(k+1)}\right]
$$

We first explain how, assuming Theorem [2.1, we can prove Theorem 1.1. When condition (1.5) holds, it has been proved in Elgart-Erdös-Schlein-Yau [20, 22, 23, 24, 25] and Kirkpatrick-Schlein-Staffilani [36] that, as trace class operators

$$
B_{N, j, k+1} \gamma_{N}^{(k+1)} \rightarrow B_{j, k+1} \gamma^{(k+1)}\left(\text { weak }^{*}\right),
$$

uniformly in $t$. (See [36, (6.7)] or [16, (5.6)], for example.) Let $\mathcal{H}_{k}$ be the Hilbert-Schmidt operators on $L^{2}\left(\mathbb{R}^{3 k}\right)$. Recall that the test functions for weak* convergence in $\mathcal{L}_{k}^{1}$ come from $\mathcal{K}_{k}$ and the test functions for weak ${ }^{*}$ convergence in $\mathcal{H}_{k}$ come from $\mathcal{H}_{k}$. Thus the weak ${ }^{*}$ convergence (2.4) as trace class operator infers that as Hilbert-Schmidt operators,

$$
B_{N, j, k+1} \gamma_{N}^{(k+1)} \rightarrow B_{j, k+1} \gamma^{(k+1)}\left(\text { weak }^{*}\right),
$$

uniformly in $t$, because $\mathcal{H}_{k} \subset \mathcal{K}_{k}$ i.e. there are fewer test functions. Since $\mathcal{H}_{k}$ is reflexive, the above weak* convergence is no different from the weak convergence. Moreover, notice 
that $P_{\leq M}^{(k)} R^{(k)} J$ is simply another test function if $J$ is a test function, we know that as Hilbert-Schmidt operators

$$
P_{\leq M}^{(k)} R^{(k)} B_{N, j, k+1} \gamma_{N}^{(k+1)} \rightarrow P_{\leq M}^{(k)} R^{(k)} B_{j, k+1} \gamma^{(k+1)}(\text { weak })
$$

uniformly in $t$. Hence, by basic properties of weak convergence

$$
\left\|P_{\leq M}^{(k)} R^{(k)} B_{j, k+1} \gamma^{(k+1)}\right\|_{L_{T}^{1} L_{\mathbf{x}, \mathbf{x}^{\prime}}^{2}} \leqslant \liminf _{N \rightarrow \infty}\left\|P_{\leq M}^{(k)} R^{(k)} B_{N, j, k+1} \gamma_{N}^{(k+1)}(t)\right\|_{L_{T}^{1} L_{\mathbf{x}, \mathbf{x}^{\prime}}^{2}} \leqslant C^{k} .
$$

Since the above holds uniformly in $M$, we can send $M \rightarrow \infty$ and, by the monotone convergence theorem, we obtain

$$
\left\|R^{(k)} B_{j, k+1} \gamma^{(k+1)}\right\|_{L_{T}^{1} L_{\mathbf{x}, \mathbf{x}^{\prime}}^{2}} \leqslant C^{k}
$$

which is exactly the Klainerman-Machedon space-time bound (1.6). This completes the proof of Theorem 1.1, assuming Theorem 2.1.

The rest of this paper is devoted to proving Theorem 2.1. We are going to establish estimate (2.2) for a sufficiently small $T$ which depends on the controlling constant in condition (1.5) and is independent of $k, N$ and $M$, then a bootstrap argument together with condition (1.5) give estimate (2.2) for every finite time at the price of a larger constant $C$. Before we start, alert readers should keep in mind that, we will mostly use the following form of condition (1.5):

$$
\left\|S^{(k)} \gamma_{N}^{(k)}\right\|_{L_{t}^{\infty} L_{\mathbf{x}, \mathbf{x}^{\prime}}^{2}} \leqslant C_{0}^{k}
$$

where $S^{(k)}=\prod_{j=1}^{k}\left(\left\langle\nabla_{x_{j}}\right\rangle\left\langle\nabla_{x_{j}^{\prime}}\right\rangle\right)$, because we will be working in $L^{2}$. To see how (2.5) follows from (1.5), one simply notices that

$$
\begin{aligned}
& \int\left|\left\langle\nabla_{x}\right\rangle\left\langle\nabla_{x^{\prime}}\right\rangle \int \phi(x, r) \overline{\phi\left(x^{\prime}, r\right)} d r\right|^{2} d x d x^{\prime} \\
= & \int\left|\int\left\langle\nabla_{x}\right\rangle \phi(x, r) \overline{\left\langle\nabla_{x^{\prime}}\right\rangle \phi\left(x^{\prime}, r\right)} d r\right|^{2} d x d x^{\prime} \\
\leqslant & \int\left(\int\left\langle\nabla_{x}\right\rangle \phi(x, r) \overline{\left\langle\nabla_{x}\right\rangle \phi(x, r)} d r\right)\left(\int\left\langle\nabla_{x^{\prime}}\right\rangle \phi\left(x^{\prime}, r\right) \overline{\left\langle\nabla_{x^{\prime}}\right\rangle \phi\left(x^{\prime}, r\right)} d r\right) d x d x^{\prime} \\
= & \left(\int \phi(x, r) \overline{\left(1-\triangle_{x}\right) \phi(x, r)} d x d r\right)^{2} .
\end{aligned}
$$

We start the proof of Theorem 2.1 by rewriting hierarchy (1.3) as

$$
\begin{aligned}
\gamma_{N}^{(k)}\left(t_{k}\right)= & U^{(k)}\left(t_{k}\right) \gamma_{N, 0}^{(k)}+\int_{0}^{t_{k}} U^{(k)}\left(t_{k}-t_{k+1}\right) V_{N}^{(k)} \gamma_{N}^{(k)}\left(t_{k+1}\right) d t_{k+1} \\
& +\frac{N-k}{N} \int_{0}^{t_{k}} U^{(k)}\left(t_{k}-t_{k+1}\right) B_{N}^{(k+1)} \gamma_{N}^{(k+1)}\left(t_{k+1}\right) d t_{k+1}
\end{aligned}
$$


with the short-hand notation:

$$
\begin{aligned}
U^{(k)} & =e^{i t \triangle_{\mathbf{x}_{k}}} e^{-i t \triangle_{\mathbf{x}_{k}^{\prime}}} \\
V_{N}^{(k)} \gamma_{N}^{(k)} & =\frac{1}{N} \sum_{1 \leqslant i<j \leqslant k}\left[V_{N}\left(x_{i}-x_{j}\right), \gamma_{N}^{(k)}\right] \\
B_{N}^{(k+1)} \gamma_{N}^{(k+1)} & =\sum_{j=1}^{k} B_{N, j, k+1} \gamma_{N}^{(k+1)} .
\end{aligned}
$$

We omit the $i$ in front of the potential term and the interaction term so that we do not need to keep track of its exact power.

Writing out the $l_{c}$ th Duhamel-Born series of $\gamma_{N}^{(k)}$ by iterating hierarchy (2.6) $l_{c}$ times 5 , we have

$$
\begin{aligned}
\gamma_{N}^{(k)}\left(t_{k}\right)= & U^{(k)}\left(t_{k}\right) \gamma_{N, 0}^{(k)} \\
& +\frac{N-k}{N} \int_{0}^{t_{k}} U^{(k)}\left(t_{k}-t_{k+1}\right) B_{N}^{(k+1)} U^{(k+1)}\left(t_{k+1}\right) \gamma_{N, 0}^{(k+1)} d t_{k+1} \\
& +\int_{0}^{t_{k}} U^{(k)}\left(t_{k}-t_{k+1}\right) V_{N}^{(k)} \gamma_{N}^{(k)}\left(t_{k+1}\right) d t_{k+1} \\
& +\frac{N-k}{N} \int_{0}^{t_{k}} U^{(k)}\left(t_{k}-t_{k+1}\right) B_{N}^{(k+1)} \\
& \times \int_{0}^{t_{k+1}} U^{(k+1)}\left(t_{k+1}-t_{k+2}\right) V_{N}^{(k+1)} \gamma_{N}^{(k+1)}\left(t_{k+2}\right) d t_{k+2} d t_{k+1} \\
& +\frac{N-k}{N} \frac{N-k-1}{N} \int_{0}^{t_{k}} U^{(k)}\left(t_{k}-t_{k+1}\right) B_{N}^{(k+1)} \\
& \times \int_{0}^{t_{k+1}} U^{(k+1)}\left(t_{k+1}-t_{k+2}\right) B_{N}^{(k+2)} \gamma_{N}^{(k+2)}\left(t_{k+2}\right) d t_{k+2} d t_{k+1}
\end{aligned}
$$

After $l_{c}$ iterations

$$
\gamma_{N}^{(k)}\left(t_{k}\right)=F P^{\left(k, l_{c}\right)}\left(t_{k}\right)+P P^{\left(k, l_{c}\right)}\left(t_{k}\right)+I P^{\left(k, l_{c}\right)}\left(t_{k}\right)
$$

where the free part at coupling level $l_{c}$ is given by

$$
\begin{aligned}
& F P^{\left(k, l_{c}\right)} \\
= & U^{(k)}\left(t_{k}\right) \gamma_{N, 0}^{(k)}+ \\
& \sum_{j=1}^{l_{c}}\left(\prod_{l=0}^{j-1} \frac{N-k-l}{N}\right) \int_{0}^{t_{k}} \cdots \int_{0}^{t_{k+j-1}} U^{(k)}\left(t_{k}-t_{k+1}\right) B_{N}^{(k+1)} \cdots \\
& \times U^{(k+j-1)}\left(t_{k+j-1}-t_{k+j}\right) B_{N}^{(k+j)}\left(U^{(k+j)}\left(t_{k+j}\right) \gamma_{N, 0}^{(k+j)}\right) d t_{k+1} \cdots d t_{k+j},
\end{aligned}
$$

\footnotetext{
${ }^{5}$ Here, $l_{c}$ stands for "level of coupling" or "length/depth of coupling". When $l_{c}=0$, we have (2.6) back.
} 
the potential part is given by

$$
\begin{aligned}
& P P^{\left(k, l_{c}\right)} \\
= & \int_{0}^{t_{k}} U^{(k)}\left(t_{k}-t_{k+1}\right) V_{N}^{(k)} \gamma_{N}^{(k)}\left(t_{k+1}\right) d t_{k+1}+\sum_{j=1}^{l_{c}}\left(\prod_{l=0}^{j-1} \frac{N-k-l}{N}\right) \\
& \times \int_{0}^{t_{k}} \cdots \int_{0}^{t_{k+j-1}} U^{(k)}\left(t_{k}-t_{k+1}\right) B_{N}^{(k+1)} \cdots U^{(k+j-1)}\left(t_{k+j-1}-t_{k+j}\right) B_{N}^{(k+j)} \\
& \times\left(\int_{0}^{t_{k+j}} U^{(k+j)}\left(t_{k+j}-t_{k+j+1}\right) V_{N}^{(k+j)} \gamma_{N}^{(k+j)}\left(t_{k+j+1}\right) d t_{k+j+1}\right) d t_{k+1} \cdots d t_{k+j},
\end{aligned}
$$

and the interaction part is given by

$$
\begin{aligned}
I P^{\left(k, l_{c}\right)} & \left(\prod_{l=0}^{l_{c}} \frac{N-k-l}{N}\right) \int_{0}^{t_{k}} \cdots \int_{0}^{t_{k+l_{c}}} U^{(k)}\left(t_{k}-t_{k+1}\right) B_{N}^{(k+1)} \cdots \\
& \cdots U^{\left(k+l_{c}\right)}\left(t_{k+l_{c}}-t_{k+l_{c}+1}\right) B_{N}^{\left(k+l_{c}+1\right)}\left(\gamma_{N}^{\left(k+l_{c}+1\right)}\left(t_{k+l_{c}+1}\right)\right) d t_{k+1} \cdots d t_{k+l_{c}+1} .
\end{aligned}
$$

By (2.7), to establish (2.2), it suffices to prove that

$$
\begin{gathered}
\left\|P_{\leq M}^{(k-1)} R^{(k-1)} B_{N, 1, k} F P^{\left(k, l_{c}\right)}\right\|_{L_{T}^{1} L_{\mathbf{x}, \mathbf{x}^{\prime}}^{2}} \leqslant C^{k-1} \\
\left\|P_{\leq M}^{(k-1)} R^{(k-1)} B_{N, 1, k} P P^{\left(k, l_{c}\right)}\right\|_{L_{T}^{1} L_{\mathbf{x}, \mathbf{x}^{\prime}}^{2}} \leqslant C^{k-1} \\
\left\|P_{\leq M}^{(k-1)} R^{(k-1)} B_{N, 1, k} I P^{\left(k, l_{c}\right)}\right\|_{L_{T}^{1} L_{\mathbf{x}, \mathbf{x}^{\prime}}^{2}} \leqslant C^{k-1}
\end{gathered}
$$

for all $k \geqslant 2$ and for some $C$ and a sufficiently small $T$ determined by the controlling constant in condition (2.5) and independent of $k, N$ and $M$. We observe that $B_{N}^{(j)}$ has $2 j$ terms inside so that each summand of $\gamma_{N}^{(k)}\left(t_{k}\right)$ contains factorially many terms $\left(\sim \frac{\left(k+l_{c}\right) !}{k !}\right)$. We use the Klainerman-Machedon board game to combine them and hence reduce the number of terms that need to be treated. Define

$$
J_{N}^{(k, j)}\left(\underline{t}_{k+j}\right)\left(f^{(k+j)}\right)=U^{(k)}\left(t_{k}-t_{k+1}\right) B_{N}^{(k+1)} \cdots U^{(k+j-1)}\left(t_{k+j-1}-t_{k+j}\right) B_{N}^{(k+j)} f^{(k+j)},
$$

where $\underline{t}_{k+j}$ means $\left(t_{k+1}, \ldots, t_{k+j}\right)$, then the Klainerman-Machedon board game implies the lemma.

Lemma 2.1 (Klainerman-Machedon board game). One can express

$$
\int_{0}^{t_{k}} \cdots \int_{0}^{t_{k+j-1}} J_{N}^{(k, j)}\left(\underline{t}_{k+j}\right)\left(f^{(k+j)}\right) d \underline{t}_{k+j}
$$

as a sum of at most $4^{j-1}$ terms of the form

$$
\int_{D} J_{N}^{(k, j)}\left(\underline{t}_{k+j}, \mu_{m}\right)\left(f^{(k+j)}\right) d \underline{t}_{k+j},
$$


or in other words,

$$
\int_{0}^{t_{k}} \cdots \int_{0}^{t_{k+j-1}} J_{N}^{(k, j)}\left(\underline{t}_{k+j}\right)\left(f^{(k+j)}\right) d \underline{t}_{k+j}=\sum_{m} \int_{D} J_{N}^{(k, j)}\left(\underline{t}_{k+j}, \mu_{m}\right)\left(f^{(k+j)}\right) d \underline{t}_{k+j} .
$$

Here $D \subset\left[0, t_{k}\right]^{j}, \mu_{m}$ are a set of maps from $\{k+1, \ldots, k+j\}$ to $\{k, \ldots, k+j-1\}$ satisfying $\mu_{m}(k+1)=k$ and $\mu_{m}(l)<l$ for all $l$, and

$$
\begin{aligned}
& J_{N}^{(k, j)}\left(\underline{t}_{k+j}, \mu_{m}\right)\left(f^{(k+j)}\right) \\
= & U^{(k)}\left(t_{k}-t_{k+1}\right) B_{N, k, k+1} U^{(k+1)}\left(t_{k+1}-t_{k+2}\right) B_{N, \mu_{m}(k+2), k+2} \cdots \\
& \cdots U^{(k+j-1)}\left(t_{k+j-1}-t_{k+j}\right) B_{N, \mu_{m}(k+j), k+j}\left(f^{(k+j)}\right) .
\end{aligned}
$$

Proof. Lemma 2.1 follows the exact same proof as [38, Theorem 3.4], the KlainermanMachedon board game, if one replaces $B_{j, k+1}$ by $B_{N, j, k+1}$ and notices that $B_{N, j, k+1}$ still commutes with $e^{i t \triangle_{x_{i}}} e^{-i t \triangle_{x_{i}^{\prime}}}$ whenever $i \neq j$. This argument reduces the number of terms by combining them.

In the rest of this paper, we establish estimate (2.9) only. The reason is the following. On the one hand, the proof of estimate (2.9) is exactly the place that relies on the restriction $\beta \in(0,2 / 3)$ in this paper. On the other hand, X.C. has already proven estimates (2.8) and (2.10) as estimates (6.3) and (6.5) in [15] without using any frequency localization. For completeness, we include a proof of estimates (2.8) and (2.10) in Appendix B. Before we delve into the proof of estimate (2.9), we remark that the proof of estimates (2.8) and (2.9) is independent of the coupling level $l_{c}$ and we will take the coupling level $l_{c}$ to be $\ln N$ for estimate (2.10) 6

\section{Estimate of the potential PART}

In this section, we prove estimate (2.9). To be specific, we establish the following theorem.

Theorem 3.1. Under the assumptions of Theorem 1.1, there exists a $C$ (independent of $\left.k, l_{c}, M_{k-1}, N\right)$ such that for each $M_{k-1} \geqslant 1$ there exists $N_{0}$ (depending on $M_{k-1}$ ) such that for $N \geqslant N_{0}$, there holds

$$
\left\|P_{\leqslant M_{k-1}}^{(k-1)} R^{(k-1)} B_{N, 1, k} P P^{\left(k, l_{c}\right)}\right\|_{L_{T}^{1} L_{\mathbf{x}, \mathbf{x}^{\prime}}^{2}} \leqslant C^{k-1}
$$

where $P P^{\left(k, l_{c}\right)}$ is given by (2.8).

In this section, we will employ the estimates stated and proved in Section 4. Due to the technicality of the proof of Theorem 3.1 involving Littlewood-Paley theory, we prove a simpler $\beta \in\left(0, \frac{2}{5}\right)$ version first to illustrate the basic steps in establishing Theorem 3.1. We then prove Theorem 3.1 in Section 3.2 .

\footnotetext{
${ }^{6}$ The technique of taking $l_{c}=\ln N$ for estimate (2.10) was first observed by T.Chen and N.Pavlović [1].
} 
3.1. A simpler proof in the case $\beta \in\left(0, \frac{2}{5}\right)$.

Theorem 3.2. For $\beta \in\left(0, \frac{2}{5}\right)$, we have the estimate

$$
\left\|R^{(k-1)} B_{N, 1, k} P P^{\left(k, l_{c}\right)}\right\|_{L_{T}^{1} L_{\mathbf{x}, \mathbf{x}^{\prime}}^{2}} \leqslant C^{k-1}
$$

for some $C$ and a sufficiently small $T$ determined by the controlling constant in condition (2.5) and independent of $k, l_{c}$ and $N$.

Proof. The proof is divided into four steps. We will reproduce every step for Theorem 3.1 in Section 3.2.

Step I. By Lemma 2.1, we know that

$$
\begin{aligned}
& \operatorname{PP}^{\left(k, l_{c}\right)} \\
= & \int_{0}^{t_{k}} U^{(k)}\left(t_{k}-t_{k+1}\right) V_{N}^{(k)} \gamma_{N}^{(k)}\left(t_{k+1}\right) d t_{k+1} \\
& +\sum_{j=1}^{l_{c}}\left(\prod_{l=0}^{j-1} \frac{N-k-l}{N}\right)\left(\sum_{m} \int_{D} J_{N}^{(k, j)}\left(\underline{t}_{k+j}, \mu_{m}\right)\left(f^{(k+j)}\right) d \underline{t}_{k+j}\right)
\end{aligned}
$$

where

$$
f^{(k+j)}=\int_{0}^{t_{k+j}} U^{(k+j)}\left(t_{k+j}-t_{k+j+1}\right) V_{N}^{(k+j)} \gamma_{N}^{(k+j)}\left(t_{k+j+1}\right) d t_{k+j+1},
$$

and the sum $\sum_{m}$ has at most $4^{j-1}$ terms inside.

For the second term in (3.1), we iterate Lemma 4.2 to prove the following estimate7:

$$
\begin{aligned}
& \left\|R^{(k-1)} B_{N, 1, k} \int_{D} J_{N}^{(k, j)}\left(\underline{t}_{k+j}, \mu_{m}\right)\left(f^{(k+j)}\right) d \underline{t}_{k+j}\right\|_{L_{T}^{1} L_{\mathbf{x}, \mathbf{x}^{\prime}}^{2}} \\
\leqslant & \left(C T^{\frac{1}{2}}\right)^{j}\left\|R^{(k+j-1)} B_{N, \mu_{m}(k+j), k+j} f^{(k+j)}\right\|_{L_{T}^{1} L_{\mathbf{x}, \mathbf{x}^{\prime}}^{2}}
\end{aligned}
$$

In fact,

$$
\begin{aligned}
& \left\|R^{(k-1)} B_{N, 1, k} \int_{D} J_{N}^{(k, j)}\left(\underline{t}_{k+j}, \mu_{m}\right)\left(f^{(k+j)}\right) d \underline{t}_{k+j}\right\|_{L_{T}^{1} L_{\mathbf{x}, \mathbf{x}^{\prime}}^{2}} \\
= & \int_{0}^{T}\left\|\int_{D} R^{(k-1)} B_{N, 1, k} U^{(k)}\left(t_{k}-t_{k+1}\right) B_{N, k, k+1} \cdots d t_{k+1} \ldots d t_{k+j}\right\|_{L_{\mathbf{x}, \mathbf{x}^{\prime}}^{2}} d t_{k} .
\end{aligned}
$$

By Minkowski,

$$
\leqslant \int_{[0, T]^{j+1}}\left\|R^{(k-1)} B_{N, 1, k} U^{(k)}\left(t_{k}-t_{k+1}\right) B_{N, k, k+1} \cdots\right\|_{L_{\mathbf{x}, \mathbf{x}^{\prime}}^{2}} d t_{k} d t_{k+1} \ldots d t_{k+j} .
$$

Cauchy-Schwarz in $d t_{k}$,

$$
\leqslant T^{\frac{1}{2}} \int_{[0, T]^{j}}\left(\int\left\|R^{(k-1)} B_{N, 1, k} U^{(k)}\left(t_{k}-t_{k+1}\right) B_{N, k, k+1} \ldots\right\|_{L_{\mathbf{x}, \mathbf{x}^{\prime}}^{2}}^{2} d t_{k}\right)^{\frac{1}{2}} d t_{k+1} \ldots d t_{k+j} .
$$

\footnotetext{
${ }^{7}$ This also helps in proving estimates (2.8) and 2.10)-see Appendix B
} 
Use Lemma 4.2,

$$
\leqslant C T^{\frac{1}{2}} \int_{[0, T]^{j}}\left\|R^{(k)} B_{N, k, k+1} U^{(k+1)}\left(t_{k+1}-t_{k+2}\right) \ldots\right\|_{L_{\mathbf{x}, \mathbf{x}^{\prime}}^{2}} d t_{k+1} \ldots d t_{k+j} .
$$

Repeat the previous steps for $j-1$ time, we then reach relation (3.3).

Applying relation (3.3) to (3.1), we have

$$
\begin{aligned}
& \left\|R^{(k-1)} B_{N, 1, k} P P^{\left(k, l_{c}\right)}\right\|_{L_{T}^{1} L_{\mathbf{x}, \mathbf{x}^{\prime}}^{2}} \\
\leqslant & \left\|R^{(k-1)} B_{N, 1, k} \int_{0}^{t_{k}} U^{(k)}\left(t_{k}-t_{k+1}\right) V_{N}^{(k)} \gamma_{N}^{(k)}\left(t_{k+1}\right) d t_{k+1}\right\|_{L_{T}^{1} L_{\mathbf{x}, \mathbf{x}^{\prime}}^{2}} \\
& +\sum_{j=1}^{l_{c}} 4^{j-1}\left(C T^{\frac{1}{2}}\right)^{j}\left\|R^{(k+j-1)} B_{N, \mu_{m}(k+j), k+j}\left(f^{(k+j)}\right)\right\|_{L_{T}^{1} L_{\mathbf{x}, \mathbf{x}^{\prime}}^{2}} \\
\leqslant & \left\|R^{(k-1)} B_{N, 1, k} \int_{0}^{t_{k}} U^{(k)}\left(t_{k}-t_{k+1}\right) V_{N}^{(k)} \gamma_{N}^{(k)}\left(t_{k+1}\right) d t_{k+1}\right\|_{L_{T}^{1} L_{\mathbf{x}, \mathbf{x}^{\prime}}^{2}} \\
& +\sum_{j=1}^{l_{c}}\left(C T^{\frac{1}{2}}\right)^{j}\left\|R^{(k+j-1)} B_{N, \mu_{m}(k+j), k+j}\left(f^{(k+j)}\right)\right\|_{L_{T}^{1} L_{\mathbf{x}, \mathbf{x}^{\prime}}^{2}} .
\end{aligned}
$$

Inserting a smooth cut-off $\theta(t)$ with $\theta(t)=1$ for $t \in[-T, T]$ and $\theta(t)=0$ for $t \in[-2 T, 2 T]^{c}$ into the above estimate, we get

$$
\begin{aligned}
& \left\|R^{(k-1)} B_{N, 1, k} P P^{\left(k, l_{c}\right)}\right\|_{L_{T}^{1} L_{\mathbf{x}, \mathbf{x}^{\prime}}^{2}} \\
\leqslant & \left\|R^{(k-1)} B_{N, 1, k} \theta\left(t_{k}\right) \int_{0}^{t_{k}} U^{(k)}\left(t_{k}-t_{k+1}\right) \theta\left(t_{k+1}\right) V_{N}^{(k)} \gamma_{N}^{(k)}\left(t_{k+1}\right) d t_{k+1}\right\|_{L_{T}^{1} L_{\mathbf{x}, \mathbf{x}^{\prime}}^{2}} \\
& +\sum_{j=1}^{l_{c}}\left(C T^{\frac{1}{2}}\right)^{j}\left\|R^{(k+j-1)} B_{N, \mu_{m}(k+j), k+j} \theta\left(t_{k+j}\right)\left(\tilde{f}^{(k+j)}\right)\right\|_{L_{T}^{1} L_{\mathbf{x}, \mathbf{x}^{\prime}}^{2}}
\end{aligned}
$$

where

$$
\tilde{f}^{(k+j)}=\int_{0}^{t_{k+j}} U^{(k+j)}\left(t_{k+j}-t_{k+j+1}\right)\left(\theta\left(t_{k+j+1}\right) V_{N}^{(k+j)} \gamma_{N}^{(k+j)}\left(t_{k+j+1}\right)\right) d t_{k+j+1}
$$

Step II. The $X_{b}$ space version of Lemma 4.2, Lemma 4.3, then turns the last step into

$$
\begin{aligned}
& \left\|R^{(k-1)} B_{N, 1, k} P P^{\left(k, l_{c}\right)}\right\|_{L_{T}^{1} L_{\mathbf{x}, \mathbf{x}^{\prime}}^{2}} \\
\leqslant & C T^{\frac{1}{2}}\left\|\theta\left(t_{k}\right) \int_{0}^{t_{k}} U^{(k)}\left(t_{k}-t_{k+1}\right) R^{(k)}\left(\theta\left(t_{k+1}\right) V_{N}^{(k)} \gamma_{N}^{(k)}\left(t_{k+1}\right)\right) d t_{k+1}\right\|_{X_{\frac{1}{2}+}^{(k)}} \\
& +C \sum_{j=1}^{l_{c}}\left(C T^{\frac{1}{2}}\right)^{j+1}\left\|\theta\left(t_{k+j}\right) R^{(k+j)} \tilde{f}^{(k+j)}\right\|_{X_{\frac{1}{2}+}^{(k+j)}}
\end{aligned}
$$


Step III. Recall the definition of $\tilde{f}^{(k+j)}$,

$$
\tilde{f}^{(k+j)}=\int_{0}^{t_{k+j}} U^{(k+j)}\left(t_{k+j}-t_{k+j+1}\right)\left(\theta\left(t_{k+j+1}\right) V_{N}^{(k+j)} \gamma_{N}^{(k+j)}\left(t_{k+j+1}\right)\right) d t_{k+j+1}
$$

so

$$
\begin{aligned}
& R^{(k+j)} \tilde{f}^{(k+j)} \\
= & \int_{0}^{t_{k+j}} U^{(k+j)}\left(t_{k+j}-t_{k+j+1}\right) R^{(k+j)}\left(\theta\left(t_{k+j+1}\right) V_{N}^{(k+j)} \gamma_{N}^{(k+j)}\left(t_{k+j+1}\right)\right) d t_{k+j+1} .
\end{aligned}
$$

We then proceed with Lemma 4.1 to get

$$
\begin{aligned}
& \left\|R^{(k-1)} B_{N, 1, k} P P^{\left(k, l_{c}\right)}\right\|_{L_{T}^{1} L_{\mathbf{x}, \mathbf{x}^{\prime}}^{2}} \\
\leqslant & C T^{\frac{1}{2}}\left\|R^{(k)}\left(\theta\left(t_{k+1}\right) V_{N}^{(k)} \gamma_{N}^{(k)}\left(t_{k+1}\right)\right)\right\|_{X_{-\frac{1}{2}+}^{(k)}} \\
& +C \sum_{j=1}^{l_{c}}\left(C T^{\frac{1}{2}}\right)^{j+1}\left\|R^{(k+j)}\left(\theta\left(t_{k+j+1}\right) V_{N}^{(k+j)} \gamma_{N}^{(k+j)}\left(t_{k+j+1}\right)\right)\right\|_{X_{-\frac{1}{2}+}^{(k+j)}} .
\end{aligned}
$$

Step IV. Now we would like to utilize Lemma 4.6. We first analyse a typical term to demonstrated the effect of Lemma 4.6. To be specific, we have

$$
\begin{aligned}
& \left\|R^{(k)}\left(\theta\left(t_{k+1}\right) V_{N}\left(x_{1}-x_{2}\right) \gamma_{N}^{(k)}\left(t_{k+1}\right)\right)\right\|_{X_{-\frac{1}{2}+}^{(k)}} \\
\leqslant & \frac{C}{N}\left\|V_{N}\left(x_{1}-x_{2}\right) \theta\left(t_{k+1}\right) R^{(k)} \gamma_{N}^{(k)}\left(t_{k+1}\right)\right\|_{X_{-\frac{1}{2}+}^{(k)}} \\
+ & \frac{C}{N}\left\|\left(V_{N}\right)^{\prime}\left(x_{1}-x_{2}\right) \theta\left(t_{k+1}\right)\left(\frac{R^{(k)}}{\left|\nabla_{x_{1}}\right|}\right) \gamma_{N}^{(k)}\left(t_{k+1}\right)\right\|_{X_{-\frac{1}{2}+}^{(k)}} \\
+ & \frac{C}{N}\left\|\left(V_{N}\right)^{\prime \prime}\left(x_{1}-x_{2}\right) \theta\left(t_{k+1}\right)\left(\frac{R^{(k)}}{\left|\nabla_{x_{1}}\right|\left|\nabla_{x_{2}}\right|}\right) \gamma_{N}^{(k)}\left(t_{k+1}\right)\right\|_{X_{-\frac{1}{2}+}^{(k)}} .
\end{aligned}
$$

by Leibniz's rule, where

$$
\frac{R^{(k)}}{\left|\nabla_{x_{1}}\right|}=\left(\prod_{j=2}^{k}\left|\nabla_{x_{j}}\right|\right)\left(\prod_{j=1}^{k}\left|\nabla_{x_{j}^{\prime}}\right|\right) .
$$

Utilize Lemma 4.6 to each summand of the above, we have

$$
\begin{aligned}
\leqslant & \frac{C}{N}\left\|V_{N}\right\|_{L^{3+}}\left\|\theta\left(t_{k+1}\right) R^{(k)} \gamma_{N}^{(k)}\right\|_{L_{t_{k+1}}^{2} L_{x, x^{\prime}}^{2}} \\
& +\frac{C}{N}\left\|V_{N}^{\prime}\right\|_{L^{2+}}\left\|\theta\left(t_{k+1}\right)\left\langle\nabla_{x_{1}}\right\rangle^{\frac{1}{2}}\left(\frac{R^{(k)}}{\left|\nabla_{x_{1}}\right|}\right) \gamma_{N}^{(k)}\right\|_{L_{t_{k+1}}^{2} L_{x, x^{\prime}}^{2}} \\
& +\frac{C}{N}\left\|V_{N}^{\prime \prime}\right\|_{L^{\frac{6}{5}+}}\left\|\theta\left(t_{k+1}\right)\left\langle\nabla_{x_{1}}\right\rangle\left\langle\nabla_{x_{2}}\right\rangle\left(\frac{R^{(k)}}{\left|\nabla_{x_{1}}\right|\left|\nabla_{x_{2}}\right|}\right) \gamma_{N}^{(k)}\right\|_{L_{t_{k+1}}^{2} L_{x, x^{\prime}}^{2}} \\
\leqslant & C\left\|S^{(k)} \gamma_{N}^{(k)}\right\|_{L_{2 T}^{2} L_{\mathbf{x}, \mathbf{x}^{\prime}}^{2}},
\end{aligned}
$$


i.e.

$$
\left\|R^{(k)}\left(\theta\left(t_{k+1}\right) V_{N}\left(x_{1}-x_{2}\right) \gamma_{N}^{(k)}\left(t_{k+1}\right)\right)\right\|_{X_{-\frac{1}{2}+}^{(2)}} \leqslant C\left\|S^{(k)} \gamma_{N}^{(k)}\right\|_{L_{2 T}^{2} L_{\mathbf{x}, \mathbf{x}^{\prime}}^{2}}
$$

since $\left\|V_{N} / N\right\|_{L^{3+}}\left\|V_{N}^{\prime} / N\right\|_{L^{2+}}$, and $\left\|V_{N}^{\prime \prime} / N\right\|_{L^{\frac{6}{5}}}$ are uniformly bounded in $N$ for $\beta \in\left(0, \frac{2}{5}\right)$. In fact,

$$
\begin{aligned}
\left\|V_{N} / N\right\|_{L^{3+}} & \leqslant N^{2 \beta-1}\|V\|_{L^{3+}} \\
\left\|V_{N}^{\prime} / N\right\|_{L^{2+}} & \leqslant N^{\frac{5 \beta}{2}-1}\left\|V^{\prime}\right\|_{L^{2+}} \\
\left\|V_{N}^{\prime \prime} / N\right\|_{L^{\frac{6}{5}+}} & \leqslant N^{\frac{5 \beta}{2}-1}\left\|V^{\prime \prime}\right\|_{L^{\frac{6}{5}+}}
\end{aligned}
$$

where by Sobolev, $V \in W^{2, \frac{6}{5}+}$ implies $V \in L^{\frac{6}{5}+} \cap L^{6+}$ and $V^{\prime} \in L^{2+}$.

Using the same idea for all the terms, we end up with

$$
\begin{aligned}
& \left\|R^{(k-1)} B_{N, 1, k} P P^{\left(k, l_{c}\right)}\right\|_{L_{T}^{1} L_{\mathbf{x}, \mathbf{x}^{\prime}}^{2}} \\
\leqslant & C T k^{2}\left\|S^{(k)} \gamma_{N}^{(k)}\right\|_{L_{2 T}^{\infty} L_{\mathbf{x}, \mathbf{x}^{\prime}}^{2}}+C T^{\frac{1}{2}} \sum_{j=1}^{l_{c}}\left(C T^{\frac{1}{2}}\right)^{j+1}(k+j)^{2}\left\|S^{(k+j)} \gamma_{N}^{(k+j)}\right\|_{L_{2 T}^{\infty} L_{\mathbf{x}, \mathbf{x}^{\prime}}^{2}}
\end{aligned}
$$

because there are $k^{2}$ terms inside $V_{N}^{(k)}$. Plug in Condition (2.5),

$$
\begin{aligned}
& \leqslant C T k^{2} C_{0}^{k}+C T^{\frac{1}{2}} \sum_{j=1}^{\infty}\left(C T^{\frac{1}{2}}\right)^{j+1}(k+j)^{2} C_{0}^{k+j} \\
& \leqslant C_{0}^{k}\left(C T k^{2}+C T^{\frac{1}{2}} k^{2} \sum_{j=1}^{\infty}\left(C T^{\frac{1}{2}}\right)^{j+1} C_{0}^{j}+C T^{\frac{1}{2}} \sum_{j=1}^{\infty}\left(C T^{\frac{1}{2}}\right)^{j+1} j^{2} C_{0}^{j}\right) .
\end{aligned}
$$

We can then choose a $T$ independent of $k, l_{c}$ and $N$ such that the two infinite series converge. We then have

$$
\begin{aligned}
\left\|R^{(k-1)} B_{N, 1, k} P P^{\left(k, l_{c}\right)}\right\|_{L_{T}^{1} L_{\mathbf{x}, \mathbf{x}^{\prime}}^{2}} & \leqslant C_{0}^{k}\left(C T k^{2}+C T^{\frac{1}{2}} k^{2}+C T^{\frac{1}{2}}\right) \\
& \leqslant C_{0}^{k}\left(C T 2^{k}+C T^{\frac{1}{2}} 2^{k}+C T^{\frac{1}{2}}\right) \\
& \leqslant C^{k-1}
\end{aligned}
$$

for some $C$ larger than $C_{0}$ because $k \geqslant 2$. This concludes the proof of Theorem 3.2 .

3.2. The case $\beta \in\left(0, \frac{2}{3}\right)$. To make formulas shorter, let us write

$$
R_{\leqslant M_{k}}^{(k)}=P_{\leqslant M_{k}}^{(k)} R^{(k)}
$$

since $P_{\leqslant M_{k}}^{(k)}$ and $R^{(k)}$ are usually bundled together. 
3.2.1. Step I. By (3.1),

$$
\begin{aligned}
& \left\|R_{\leqslant M_{k-1}}^{(k-1)} B_{N, 1, k} P P^{\left(k, l_{c}\right)}\right\|_{L_{T}^{1} L_{\mathbf{x}, \mathbf{x}^{\prime}}^{2}} \\
\leqslant & \left\|R_{\leqslant M_{k-1}}^{(k-1)} B_{N, 1, k} \int_{0}^{t_{k}} U^{(k)}\left(t_{k}-t_{k+1}\right) V_{N}^{(k)} \gamma_{N}^{(k)}\left(t_{k+1}\right) d t_{k+1}\right\|_{L_{T}^{1} L_{\mathbf{x}, \mathbf{x}^{\prime}}^{2}} \\
& +\sum_{j=1}^{l_{c}} \sum_{m}\left\|R_{\leqslant M_{k-1}}^{(k-1)} B_{N, 1, k} \int_{D} J_{N}^{(k, j)}\left(\underline{t}_{k+j}, \mu_{m}\right)\left(f^{(k+j)}\right) d \underline{t}_{k+j}\right\|_{L_{T}^{1} L_{\mathbf{x}, \mathbf{x}^{\prime}}^{2}}
\end{aligned}
$$

where $f^{(k+j)}$ is again given by $(3.2)$ and the sum $\sum_{m}$ has at most $4^{j-1}$ terms inside. By Minkowski's integral inequality,

$$
\begin{aligned}
& \left\|R_{\leqslant M_{k-1}}^{(k-1)} B_{N, 1, k} \int_{D} J_{N}^{(k, j)}\left(\underline{t}_{k+j}, \mu_{m}\right)\left(f^{(k+j)}\right) d \underline{t}_{k+j}\right\|_{L_{T}^{1} L_{\mathbf{x}, \mathbf{x}^{\prime}}^{2}}\left\|\underline{t}_{k+j}\right\|_{L_{\mathbf{x}, \mathbf{x}^{\prime}}^{2}} d t_{k} \\
= & \int_{0}^{T} d t_{k}\left\|\int_{D} R_{\leqslant M_{k-1}}^{(k-1)} B_{N, 1, k} J_{N}^{(k, j)}\left(\underline{t}_{k+j}, \mu_{m}\right)\left(f^{(k+j)}\right) d\right\|_{L_{\mathbf{x}, \mathbf{x}^{\prime}}} d t_{k} d \underline{t}_{k+j} \\
\leqslant & \int_{[0, T]^{j+1}}\left\|R_{\leqslant M_{k-1}}^{(k-1)} B_{N, 1, k} U^{(k)}\left(t_{k}-t_{k+1}\right) B_{N, k, k+1} \cdots\right\|^{2}
\end{aligned}
$$

By Cauchy-Schwarz in the $t_{k}$ integration,

$$
\leqslant T^{\frac{1}{2}} \int_{[0, T]^{j}}\left(\int\left\|R_{\leqslant M_{k-1}}^{(k-1)} B_{N, 1, k} U^{(k)}\left(t_{k}-t_{k+1}\right) B_{N, k, k+1} \cdots\right\|_{L_{\mathbf{x}, \mathbf{x}^{\prime}}^{2}} d t_{k}\right)^{\frac{1}{2}} d \underline{t}_{k+j}
$$

By Lemma 4.4,

$$
\leqslant C_{\varepsilon} T^{\frac{1}{2}} \sum_{M_{k} \geqslant M_{k-1}}\left(\frac{M_{k-1}}{M_{k}}\right)^{1-\varepsilon} \int_{[0, T]^{j}}\left\|R_{\leqslant M_{k}}^{(k)} B_{N, k, k+1} U^{(k+1)}\left(t_{k+1}-t_{k+2}\right) \cdots\right\|_{L_{\mathbf{x}, \mathbf{x}^{\prime}}^{2}} d \underline{t}_{k+j}
$$

Iterating the previous step $(j-1)$ times,

$$
\begin{aligned}
\leqslant & \left(C_{\varepsilon} T^{\frac{1}{2}}\right)^{j} \sum_{M_{k+j-1} \geqslant \cdots \geqslant M_{k} \geqslant M_{k-1}}\left[\left(\frac{M_{k-1}}{M_{k}} \frac{M_{k}}{M_{k+1}} \cdots \frac{M_{k+j-2}}{M_{k+j-1}}\right)^{1-\varepsilon}\right. \\
& \left.\times\left\|R_{\leqslant M_{k+j-1}}^{(k+j-1)} B_{N, \mu_{m}(k+j), k+j}\left(f^{(k+j)}\right)\right\|_{L_{T}^{1} L_{\mathbf{x}, \mathbf{x}^{\prime}}^{2}}\right] \\
= & \left(C_{\varepsilon} T^{\frac{1}{2}}\right)^{j} \sum_{M_{k+j-1} \geqslant \cdots \geqslant M_{k} \geqslant M_{k-1}}\left[\left(\frac{M_{k-1}}{M_{k+j-1}}\right)^{1-\varepsilon}\right. \\
& \left.\times\left\|R_{\leqslant M_{k+j-1}}^{(k+j-1)} B_{N, \mu_{m}(k+j), k+j}\left(f^{(k+j)}\right)\right\|_{L_{T}^{1} L_{\mathbf{x}, \mathbf{x}^{\prime}}^{2}}\right]
\end{aligned}
$$

where the sum is over all $M_{k}, \ldots, M_{k+j-1}$ dyadic such that $M_{k+j-1} \geqslant \cdots \geqslant M_{k} \geqslant M_{k-1}$. 
Hence

$$
\begin{aligned}
& \left\|R_{\leqslant M_{k-1}}^{(k-1)} B_{N, 1, k} P P^{\left(k, l_{c}\right)}\right\|_{L_{T}^{1} L_{\mathbf{x}, \mathbf{x}^{\prime}}^{2}} \\
\leqslant & \left\|R_{\leqslant M_{k-1}}^{(k-1)} B_{N, 1, k} \int_{0}^{t_{k}} U^{(k)}\left(t_{k}-t_{k+1}\right) V_{N}^{(k)} \gamma_{N}^{(k)}\left(t_{k+1}\right) d t_{k+1}\right\|_{L_{T}^{1} L_{\mathbf{x}, \mathbf{x}^{\prime}}^{2}} \\
& +\sum_{j=1}^{l_{c}}\left\{( C _ { \varepsilon } T ^ { \frac { 1 } { 2 } } ) ^ { j } \sum _ { M _ { k + j - 1 } \geqslant \cdots \geqslant M _ { k } \geqslant M _ { k - 1 } } \left[\frac{M_{k-1}^{1-\varepsilon}}{M_{k+j-1}^{1-\varepsilon}}\right.\right. \\
& \left.\left.\times R_{\leqslant M_{k+j-1}}^{(k+j-1)} B_{N, \mu_{m}(k+j), k+j}\left(f^{(k+j)}\right) \|_{L_{T}^{1} L_{\mathbf{x}, \mathbf{x}^{\prime}}^{2}}\right]\right\}
\end{aligned}
$$

We then insert a smooth cut-off $\theta(t)$ with $\theta(t)=1$ for $t \in[-T, T]$ and $\theta(t)=0$ for $t \in[-2 T, 2 T]^{c}$ into the above estimate to get

$$
\begin{aligned}
& \left\|R_{\leqslant M_{k-1}}^{(k-1)} B_{N, 1, k} P P^{\left(k, l_{c}\right)}\right\|_{L_{T}^{1} L_{\mathbf{x}, \mathbf{x}^{\prime}}^{2}} \\
\leqslant & \left\|R_{\leqslant M_{k-1}}^{(k-1)} B_{N, 1, k} \theta\left(t_{k}\right) \int_{0}^{t_{k}} U^{(k)}\left(t_{k}-t_{k+1}\right) \theta\left(t_{k+1}\right) V_{N}^{(k)} \gamma_{N}^{(k)}\left(t_{k+1}\right) d t_{k+1}\right\|_{L_{T}^{1} L_{\mathbf{x}, \mathbf{x}^{\prime}}^{2}} \\
& +\sum_{j=1}^{l_{c}}\left\{( C _ { \varepsilon } T ^ { \frac { 1 } { 2 } } ) ^ { j } \sum _ { M _ { k + j - 1 } \geqslant \cdots \geqslant M _ { k } \geqslant M _ { k - 1 } } \left[\frac{M_{k-1}^{1-\varepsilon}}{M_{k+j-1}^{1-\varepsilon}}\right.\right. \\
& \left.\left.\times\left\|R_{\leqslant M_{k+j-1}}^{(k+j-1)} B_{N, \mu_{m}(k+j), k+j}\left(\theta\left(t_{k+j}\right) \tilde{f}^{(k+j)}\right)\right\|_{L_{T}^{1} L_{\mathbf{x}, \mathbf{x}^{\prime}}^{2}}\right]\right\},
\end{aligned}
$$

where the sum is over all $M_{k}, \ldots, M_{k+j-1}$ dyadic such that $M_{k+j-1} \geqslant \cdots \geqslant M_{k} \geqslant M_{k-1}$, and $\tilde{f}^{(k+j)}$ is again defined via (3.4).

3.2.2. Step II. Using Lemma 4.5, the $X_{b}$ space version of Lemma 4.4, we turn Step I into

$$
\begin{aligned}
& \left\|R_{\leqslant M_{k-1}}^{(k-1)} B_{N, 1, k} P P^{\left(k, l_{c}\right)}\right\|_{L_{T}^{1} L_{\mathbf{x}, \mathbf{x}^{\prime}}^{2}} \\
& \leqslant C_{\varepsilon} T^{\frac{1}{2}} \sum_{M_{k} \geqslant M_{k-1}}\left[\frac{M_{k-1}^{1-\varepsilon}}{M_{k}^{1-\varepsilon}}\right. \\
& \left.\quad \times\left\|\int_{0}^{t_{k}} U^{(k)}\left(t_{k}-t_{k+1}\right)\left(R_{\leqslant M_{k}}^{(k)} \theta\left(t_{k+1}\right) V_{N}^{(k)} \gamma_{N}^{(k)}\left(t_{k+1}\right)\right) d t_{k+1}\right\|_{X_{\frac{1}{2}+}^{(k)}}\right] \\
& +\sum_{j=1}^{l_{c}}\left(C_{\varepsilon} T^{\frac{1}{2}}\right)^{j+1} \sum_{M_{k+j} \geqslant M_{k+j-1} \geqslant \cdots \geqslant M_{k} \geqslant M_{k-1}}\left[\frac{M_{k-1}^{1-\varepsilon}}{M_{k+j}^{1-\varepsilon}}\right. \\
& \left.\quad \times\left\|\theta\left(t_{k+j}\right) R_{\leqslant M_{k+j}}^{(k+j)}\left(\tilde{f}^{(k+j)}\right)\right\|_{X_{\frac{1}{2}+j}^{(k+j)}}\right]
\end{aligned}
$$


3.2.3. Step III. Lemma 4.1 gives us

$$
\left\|R_{\leqslant M_{k-1}}^{(k-1)} B_{N, 1, k} P P^{\left(k, l_{c}\right)}\right\|_{L_{T}^{1} L_{\mathbf{x}, \mathbf{x}^{\prime}}^{2}} \leqslant A+B
$$

where

$$
A=C_{\varepsilon} T^{\frac{1}{2}} \sum_{M_{k} \geqslant M_{k-1}} \frac{M_{k-1}^{1-\varepsilon}}{M_{k}^{1-\varepsilon}}\left\|R_{\leqslant M_{k}}^{(k)} \theta\left(t_{k+1}\right) V_{N}^{(k)} \gamma_{N}^{(k)}\left(t_{k+1}\right)\right\|_{X_{-\frac{1}{2}+}^{(k)}}
$$

and

$$
\begin{aligned}
B= & \sum_{j=1}^{l_{c}}\left\{( C _ { \varepsilon } T ^ { \frac { 1 } { 2 } } ) ^ { j + 1 } \sum _ { M _ { k + j } \geqslant M _ { k + j - 1 } \geqslant \cdots \geqslant M _ { k } \geqslant M _ { k - 1 } } \left[\frac{M_{k-1}^{1-\varepsilon}}{M_{k+j}^{1-\varepsilon}}\right.\right. \\
& \left.\left.\times\left\|R_{\leqslant M_{k+j}}^{(k+j)} \theta\left(t_{k+j+1}\right) V_{N}^{(k+j)} \gamma_{N}^{(k+j)}\left(t_{k+j+1}\right)\right\|_{X_{-\frac{1}{2}+}^{(k+j)}}\right]\right\}
\end{aligned}
$$

3.2.4. Step $I V$. We focus for a moment on $B$. First, we carry out the sum in $M_{k} \leqslant \cdots \leqslant$ $M_{k+j-1}$ with the help of Lemma 3.1

$$
\begin{aligned}
B= & \sum_{j=1}^{l_{c}}\left\{( C _ { \varepsilon } T ^ { \frac { 1 } { 2 } } ) ^ { j + 1 } \sum _ { M _ { k + j } \geqslant M _ { k - 1 } } \left[\frac{M_{k-1}^{1-\varepsilon}}{M_{k+j}^{1-\varepsilon}}\left(\frac{\left(\log _{2} \frac{M_{k+j}}{M_{k-1}}+j\right)^{j}}{j !}\right)\right.\right. \\
& \left.\left.\times\left\|R_{\leqslant M_{k+j}}^{(k+j)}\left(\theta\left(t_{k+j+1}\right) V_{N}^{(k+j)} \gamma_{N}^{(k+j)}\left(t_{k+j+1}\right)\right)\right\|_{X_{-\frac{1}{2}+}^{(k+j)}}\right]\right\}
\end{aligned}
$$

We then take a $T^{j / 4}$ from the front to apply Lemma 3.2 and get to

$$
\begin{aligned}
B \lesssim & C_{\varepsilon} T^{\frac{1}{2}} \sum_{j=1}^{l_{c}}\left\{( C _ { \varepsilon } T ^ { \frac { 1 } { 4 } } ) ^ { j } \sum _ { M _ { k + j } \geqslant M _ { k - 1 } } \left[\frac{M_{k-1}^{1-2 \varepsilon}}{M_{k+j}^{1-2 \varepsilon}}\right.\right. \\
& \left.\left.\times\left\|R_{\leqslant M_{k+j}}^{(k+j)}\left(\theta\left(t_{k+j+1}\right) V_{N}^{(k+j)} \gamma_{N}^{(k+j)}\left(t_{k+j+1}\right)\right)\right\|_{X_{-\frac{1}{2}+}^{(k+j)}}\right]\right\}
\end{aligned}
$$

where the sum is over dyadic $M_{k+j}$ such that $M_{k+j} \geqslant M_{k-1}$. Applying (4.26),

$$
\begin{aligned}
B \lesssim & C_{\varepsilon} T^{\frac{1}{2}} \sum_{j=1}^{l_{c}}\left\{( C _ { \varepsilon } T ^ { \frac { 1 } { 4 } } ) ^ { j } ( k + j ) ^ { 2 } \sum _ { M _ { k + j } \geqslant M _ { k - 1 } } \left[\frac{M_{k-1}^{1-2 \varepsilon}}{M_{k+j}^{1-2 \varepsilon}} \min \left(M_{k+j}^{2}, N^{2 \beta}\right) N^{\frac{1}{2} \beta-1}\right.\right. \\
& \left.\left.\times\left\|\theta\left(t_{k+j+1}\right) S^{(k+j)} \gamma_{N}^{(k+j)}\left(t_{k+j+1}\right)\right\|_{L_{t_{k+j+1}}^{2} L_{\mathbf{x}, \mathbf{x}^{\prime}}^{2}}\right]\right\}
\end{aligned}
$$

Rearranging terms

$$
\begin{aligned}
B \lesssim & C_{\varepsilon} T^{\frac{1}{2}} \sum_{j=1}^{l_{c}}\left\{\left(C_{\varepsilon} T^{\frac{1}{4}}\right)^{j}(k+j)^{2}\left\|\theta\left(t_{k+j+1}\right) S^{(k+j)} \gamma_{N}^{(k+j)}\left(t_{k+j+1}\right)\right\|_{L_{t_{k+j+1}^{2}}^{2} L_{\mathbf{x}, \mathbf{x}^{\prime}}^{2}}\right. \\
& \left.\times M_{k-1}^{1-2 \epsilon} N^{\frac{1}{2} \beta-1} \sum_{M_{k+j} \geqslant M_{k-1}}(\cdots)\right\}
\end{aligned}
$$


where

$$
\sum_{M_{k+j} \geqslant M_{k-1}}(\cdots)=\sum_{M_{k+j} \geqslant M_{k-1}} \min \left(M_{k+j}^{1+2 \epsilon}, M_{k+j}^{-1+2 \epsilon} N^{2 \beta}\right) .
$$

We carry out the sum in $M_{k+j}$ by dividing into $M_{k+j} \leqslant N^{\beta}$ (for which $\min \left(M_{k+j}^{1+2 \epsilon}, M_{k+j}^{-1+2 \epsilon} N^{2 \beta}\right)=$ $M_{j}^{1+2 \epsilon}$ ) and $M_{k+j} \geqslant N^{\beta}$ (for which $\min \left(M_{k+j}^{1+2 \epsilon}, M_{k+j}^{-1+2 \epsilon} N^{2 \beta}\right)=M_{k+j}^{-1+2 \epsilon} N^{2 \beta}$ ). This yields

$$
\begin{aligned}
\sum_{M_{k+j} \geqslant M_{k-1}} \min \left(M_{k+j}^{1+2 \epsilon}, M_{k+j}^{-1+2 \epsilon} N^{2 \beta}\right) & \lesssim\left(\sum_{N^{\beta} \geqslant M_{k+j} \geqslant M_{k-1}}+\sum_{M_{k+j} \geqslant M_{k-1}, M_{k+j} \geqslant N^{\beta}}\right)(\ldots) \\
& \lesssim \sum_{N^{\beta} \geqslant M_{k+j} \geqslant 1} M_{k+j}^{1+2 \epsilon}+\sum_{M_{k+j} \geqslant N^{\beta}} M_{k+j}^{-1+2 \epsilon} N^{2 \beta} \\
& \lesssim N^{\beta+2 \epsilon} .
\end{aligned}
$$

Hence

$$
\begin{aligned}
B \lesssim & C_{\varepsilon} T^{\frac{1}{2}} \sum_{j=1}^{l_{c}}\left[\left(C_{\varepsilon} T^{\frac{1}{4}}\right)^{j}(k+j)^{2}\right. \\
& \left.\times\left\|\theta\left(t_{k+j+1}\right) S^{(k+j)} \gamma_{N}^{(k+j)}\left(t_{k+j+1}\right)\right\|_{L_{t_{k+j+1}^{2}}^{2} L_{\mathbf{x}, \mathbf{x}^{\prime}}^{2}} M_{k-1}^{1-2 \epsilon} N^{\frac{3}{2} \beta-1+2 \epsilon}\right] \\
\lesssim & M_{k-1}^{1-2 \epsilon} N^{\frac{3}{2} \beta-1+2 \epsilon} C_{\varepsilon} T^{\frac{1}{2}} \sum_{j=1}^{l_{c}}\left(C_{\varepsilon} T^{\frac{1}{4}}\right)^{j}(k+j)^{2} T^{\frac{1}{2}}\left\|S^{(k+j)} \gamma_{N}^{(k+j)}\right\|_{L_{t}^{\infty} L_{\mathbf{x}, \mathbf{x}^{\prime}}^{2}}
\end{aligned}
$$

Via Condition (2.5), it becomes

$$
\begin{aligned}
B & \lesssim M_{k-1}^{1-2 \epsilon} N^{\frac{3}{2} \beta-1+2 \epsilon} C_{\varepsilon} T \sum_{j=1}^{l_{c}}\left(C_{\varepsilon} T^{\frac{1}{4}}\right)^{j}(k+j)^{2} C_{0}^{k+j} \\
& \lesssim C_{0}^{k} M_{k-1}^{1-2 \epsilon} N^{\frac{3}{2} \beta-1+2 \epsilon} C_{\varepsilon} T\left(k^{2} \sum_{j=1}^{\infty}\left(C_{\varepsilon} T^{\frac{1}{4}}\right)^{j} C_{0}^{j}+\sum_{j=1}^{\infty}\left(C_{\varepsilon} T^{\frac{1}{4}}\right)^{j} j^{2} C_{0}^{j}\right)
\end{aligned}
$$

We can then choose a $T$ independent of $M_{k-1}, k, l_{c}$ and $N$ such that the two infinite series converge. We then have

$$
B \lesssim C^{k-1} M_{k-1}^{1-2 \epsilon} N^{\frac{3}{2} \beta-1+2 \epsilon}
$$

for some $C$ larger than $C_{0}$. Therefore, for $\beta<2 / 3$, there is a $C$ independent of $M_{k-1}, k, l_{c}$, and $N$ s.t. given a $M_{k-1}$, there is $N_{0}\left(M_{k-1}\right)$ which makes

$$
B \leqslant C^{k-1} \text {, for all } N \geqslant N_{0} .
$$

This completes the treatment of term $B$ for $\beta<2 / 3$. Term $A$ is treated similarly (without the need to appeal to Lemmas 3.1, 3.2 below). Whence we have completed the proof of Theorem 3.1 and thence Theorem 2.1.

\section{Lemma 3.1.}

$$
\left(\sum_{M_{k-1} \leq M_{k} \leq \cdots \leq M_{k+j-1} \leq M_{k+j}} 1\right) \leq \frac{\left(\log _{2} \frac{M_{k+j}}{M_{k-1}}+j\right)^{j}}{j !},
$$


where the sum is in $M_{k} \leq \cdots \leq M_{k+j-1}$ over dyads, such that $M_{k-1} \leq M_{k} \leq \cdots \leq M_{k+j-1} \leq$ $M_{k+j}$.

Proof. This is equivalent to

$$
\left(\sum_{i_{k-1} \leq i_{k} \leq \cdots \leq i_{k+j-1} \leq i_{k+j}} 1\right) \leq \frac{\left(i_{k+j}-i_{k-1}+j\right)^{j}}{j !}
$$

where the sum is taken over integers $i_{k}, \ldots, i_{k+j-1}$ such that $i_{k-1} \leq i_{k} \leq \cdots \leq i_{k+j-1} \leq i_{k+j}$. We use the estimate (for $p \geq 0, \ell \geq 0$ )

$$
\sum_{i=0}^{q}(i+\ell)^{p} \leq \frac{(q+\ell+1)^{p+1}}{p+1}
$$

which just follows by estimating the sum by an integral.

First, carry out the sum in $i_{k}$ from $i_{k-1}$ to $i_{k+1}$ to obtain

$$
=\sum_{i_{k-1} \leq i_{k+1} \leq \cdots \leq i_{k+j-1} \leq i_{k+j}}\left(\sum_{i_{k}=i_{k-1}}^{i_{k+1}} 1\right) \leq \sum_{i_{k-1} \leq i_{k+1} \leq \cdots \leq i_{k+j-1} \leq i_{k+j}}\left(i_{k+1}-i_{k-1}+1\right) .
$$

Next, carry out the sum in $i_{k+1}$ from $i_{k-1}$ to $i_{k+2}$,

$$
\begin{aligned}
& \leq \sum_{i_{k-1} \leq i_{k+2} \leq \cdots \leq i_{k+j-1} \leq i_{k+j}}\left(\sum_{i_{k+1}=i_{k-1}}^{i_{k+2}}\left(i_{k+1}-i_{k-1}+1\right)\right) \\
& \leq \sum_{i_{k-1} \leq i_{k+2} \leq \cdots \leq i_{k+j-1} \leq i_{k+j}}\left(\sum_{i_{k+1}=0}^{i_{k+2}-i_{k-1}}\left(i_{k+1}+1\right)\right) \\
& \leq \sum_{i_{k-1} \leq i_{k+2} \leq \cdots \leq i_{k+j-1} \leq i_{k+j}} \frac{\left(i_{k+2}-i_{k-1}+2\right)^{2}}{2} .
\end{aligned}
$$

Continue in this manner for $j-2$ times to obtain the claimed bound.

Lemma 3.2. For each $\alpha>0$ (possibly large) and each $\epsilon>0$ (arbitrarily small), there exists $t>0$ (independent of $M$ ) sufficiently small such that

$$
\forall j \geq 1, \forall M, \quad \text { we have } \frac{t^{j}(\alpha \log M+j)^{j}}{j !} \leq M^{\epsilon}
$$

Proof. We use the following fact: for each $\sigma>0$ (arbitrarily small) there exists $t>0$ sufficiently small such that

$$
\forall x>0, \quad t^{x}\left(\frac{1}{x}+1\right)^{x} \leq e^{\sigma}
$$

To apply this fact to prove the lemma, use Stirling's formula to obtain

$$
\frac{t^{j}(\alpha \log M+j)^{j}}{j !} \leq(e t)^{j}\left(\frac{\alpha \log M+j}{j}\right)^{j}
$$


Define $x$ in terms of $j$ by the formula $j=\alpha(\log M) x$. Then

$$
\left.=\left[(e t)^{x}\left(\frac{1}{x}+1\right)\right]^{x}\right]^{\alpha \log M}
$$

Applying (3.5),

$$
\leq e^{\sigma \alpha \log M}=M^{\sigma \alpha}
$$

\section{Collapsing and Strichartz estimates}

Define the norm

$$
\left\|\alpha^{(k)}\right\|_{X_{b}^{(k)}}=\left(\int\left\langle\tau+\left|\boldsymbol{\xi}_{k}\right|^{2}-\left|\boldsymbol{\xi}_{k}^{\prime}\right|^{2}\right\rangle^{2 b}\left|\hat{\alpha}^{(k)}\left(\tau, \boldsymbol{\xi}_{k}, \boldsymbol{\xi}_{k}^{\prime}\right)\right|^{2} d \tau d \boldsymbol{\xi}_{k} d \boldsymbol{\xi}_{k}^{\prime}\right)^{1 / 2}
$$

We will use the case $b=\frac{1}{2}+$ of the following lemma.

Lemma 4.1. Let $\frac{1}{2}<b<1$ and $\theta(t)$ be a smooth cutoff. Then

$$
\left\|\theta(t) \int_{0}^{t} U^{(k)}(t-s) \beta^{(k)}(s) d s\right\|_{X_{b}^{(k)}} \lesssim\left\|\beta^{(k)}\right\|_{X_{b-1}^{(k)}}
$$

Proof. The estimate reduces to the space-independent estimate

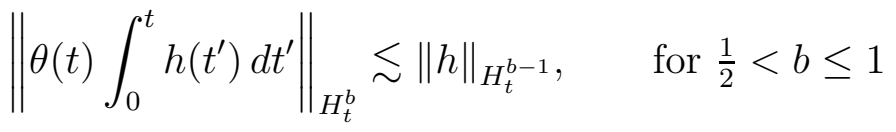

Indeed, taking $h(t)=h_{\boldsymbol{x}_{k} \boldsymbol{x}_{k}^{\prime}}(t) \stackrel{\text { def }}{=} U^{(k)}(-t) \beta^{(k)}\left(t, \boldsymbol{x}_{k}, \boldsymbol{x}_{k}^{\prime}\right)$, applying the estimate (4.2) for fixed $\mathbf{x}_{k}, \mathbf{x}_{k}^{\prime}$, and then applying the $L_{\mathbf{x}_{k} \mathbf{x}_{k}^{\prime}}^{2}$ norm to both sides, yields (4.1). Now we prove estimate (4.2). Let $P_{\leq 1}$ and $P_{\geq 1}$ denote Littlewood-Paley projections onto frequencies $|\tau| \lesssim 1$ and $|\tau| \gtrsim 1$ respectively. Decompose $h=P_{\leq 1} h+P_{\geq 1} h$ and use that $\int_{0}^{t} P_{\geq 1} h\left(t^{\prime}\right)=\frac{1}{2} \int(\operatorname{sgn}(t-$ $\left.\left.t^{\prime}\right)+\operatorname{sgn}\left(t^{\prime}\right)\right) P_{\geq 1} h\left(t^{\prime}\right) d t^{\prime}$ to obtain the decomposition

$$
\theta(t) \int_{0}^{t} h\left(t^{\prime}\right) d t^{\prime}=H_{1}(t)+H_{2}(t)+H_{3}(t)
$$

where

$$
\begin{aligned}
& H_{1}(t)=\theta(t) \int_{0}^{t} P_{\leq 1} h\left(t^{\prime}\right) d t^{\prime} \\
& H_{2}(t)=\frac{1}{2} \theta(t)\left[\operatorname{sgn} * P_{\geq 1} h\right](t) d t^{\prime} \\
& H_{3}(t)=\frac{1}{2} \theta(t) \int_{-\infty}^{+\infty} \operatorname{sgn}\left(t^{\prime}\right) P_{\geq 1} h\left(t^{\prime}\right) d t^{\prime} .
\end{aligned}
$$

We begin by addressing term $H_{1}$. By Sobolev embedding (recall $\frac{1}{2}<b \leq 1$ ) and the $L^{p} \rightarrow L^{p}$ boundedness of the Hilbert transform for $1<p<\infty$,

$$
\left\|H_{1}\right\|_{H_{t}^{b}} \lesssim\left\|H_{1}\right\|_{L_{t}^{2}}+\left\|\partial_{t} H_{1}\right\|_{L_{t}^{2 /(3-2 b)}} .
$$

Using that $\left\|P_{\leq 1} h\right\|_{L_{t}^{\infty}} \lesssim\|h\|_{H_{t}^{b-1}}$, we thus conclude

$$
\left\|H_{1}\right\|_{H_{t}^{b}} \lesssim\left(\|\theta\|_{L_{t}^{2}}+\|\theta\|_{L_{t}^{2 /(3-2 b)}}+\left\|\theta^{\prime}\right\|_{L_{t}^{2 / 3-2 b}}\right)\|h\|_{H_{t}^{b-1}} .
$$


Next we address the term $H_{2}$. By the fractional Leibniz rule,

$$
\left\|H_{2}\right\|_{H_{t}^{b}} \lesssim\left\|\left\langle D_{t}\right\rangle^{b} \theta\right\|_{L_{t}^{2}}\left\|\operatorname{sgn} * P_{\geq 1} h\right\|_{L_{t}^{\infty}}+\|\theta\|_{L_{t}^{\infty}}\left\|\left\langle D_{t}\right\rangle^{b}\left(\operatorname{sgn} * P_{\geq 1} h\right)\right\|_{L_{t}^{2}} .
$$

However,

$$
\left\|\operatorname{sgn} * P_{\geq 1} h\right\|_{L_{t}^{\infty}} \lesssim\left\|\langle\tau\rangle^{-1} \hat{h}(\tau)\right\|_{L_{\tau}^{1}} \lesssim\|h\|_{H_{t}^{b-1}}
$$

On the other hand,

$$
\left\|\left\langle D_{t}\right\rangle^{b} \operatorname{sgn} * P_{\geq 1} h\right\|_{L_{t}^{2}} \lesssim\left\|\langle\tau\rangle^{b}\langle\tau\rangle^{-1} \hat{h}(\tau)\right\|_{L_{\tau}^{2}} \lesssim\|h\|_{H_{t}^{b-1}}
$$

Consequently,

$$
\left\|H_{2}\right\|_{H_{t}^{b}} \lesssim\left(\left\|\left\langle D_{t}\right\rangle^{b} \theta\right\|_{L_{t}^{2}}+\|\theta\|_{L_{t}^{\infty}}\right)\|h\|_{H_{t}^{b-1}}
$$

For term $H_{3}$, we have

$$
\left\|H_{3}\right\|_{H_{t}^{b}} \lesssim\|\theta\|_{H_{t}^{b}}\left\|\int_{-\infty}^{+\infty} \operatorname{sgn}\left(t^{\prime}\right) P_{\geq 1} h\left(t^{\prime}\right) d t^{\prime}\right\|_{L_{t}^{\infty}} .
$$

However, the second term is handled via Parseval's identity

$$
\int_{t^{\prime}} \operatorname{sgn}\left(t^{\prime}\right) P_{\geq 1} h\left(t^{\prime}\right) d t^{\prime}=\int_{|\tau| \geq 1} \tau^{-1} \hat{h}(\tau) d \tau,
$$

from which the appropriate bounds follow again by Cauchy-Schwarz. Collecting our estimates for $H_{1}, H_{2}$, and $H_{3}$, we have

$$
\left\|\theta(t) \int_{0}^{t} h\left(t^{\prime}\right) d t^{\prime}\right\|_{H_{t}^{b}} \lesssim C_{\theta}\|h\|_{H_{t}^{b-1}},
$$

where

$$
C_{\theta}=\|\theta\|_{L_{t}^{2}}+\left\|\theta^{\prime}\right\|_{L_{t}^{2 /(3-2 b)}}+\left\|\left\langle D_{t}\right\rangle^{b} \theta\right\|_{L_{t}^{2}}+\|\theta\|_{L_{t}^{2 /(3-2 b)}}+\|\theta\|_{L_{t}^{\infty}}
$$

\subsection{Various forms of collapsing estimates.}

Lemma 4.2. There is a $C$ independent of $j, k$, and $N$ such that, $\left(\right.$ for $f^{(k+1)}\left(\mathbf{x}_{k+1}, \mathbf{x}_{k+1}\right)$ independent of $t$ )

$$
\left\|R^{(k)} B_{N, j, k+1} U^{(k+1)}(t) f^{(k+1)}\right\|_{L_{t}^{2} L_{\mathbf{x}, \mathbf{x}^{\prime}}^{2}} \leqslant C\|V\|_{L^{1}}\left\|R^{(k+1)} f^{(k+1)}\right\|_{L_{\mathbf{x}, \mathbf{x}^{\prime}}^{2}} .
$$

Proof. One can find this estimate as estimate (A.18) in [11] or a special case of Theorem 7 of [15]. For more estimates of this type, see [36, 29, 12, 14, 3, 28].

We have the following consequence of Lemma 4.2.

Lemma 4.3. There is a $C$ independent of $j, k$, and $N$ such that $\left(\right.$ for $\alpha^{(k+1)}\left(t, \mathbf{x}_{k+1}, \mathbf{x}_{k+1}\right)$ dependent on $t$ )

$$
\left\|R^{(k)} B_{N, j, k+1} \alpha^{(k+1)}\right\|_{L_{t}^{2} L_{\mathbf{x}, \mathbf{x}^{\prime}}^{2}} \leqslant C\left\|R^{(k+1)} \alpha^{(k+1)}\right\|_{X_{\frac{1}{2}+}^{(k+1)}}
$$


Proof. Let

$$
f_{\tau}^{(k+1)}\left(\mathbf{x}_{k+1}, \mathbf{x}_{k+1}^{\prime}\right)=\mathcal{F}_{t \mapsto \tau}\left(U^{(k+1)}(-t) \alpha^{(k+1)}\left(t, \mathbf{x}_{k+1}, \mathbf{x}_{k+1}^{\prime}\right)\right)
$$

where $\mathcal{F}_{t \mapsto \tau}$ denotes the Fourier transform in $t \mapsto \tau$. Then

$$
\alpha^{(k+1)}\left(t, \mathbf{x}_{k+1}, \mathbf{x}_{k+1}^{\prime}\right)=\int_{\tau} e^{i t \tau} U^{(k+1)}(t) f^{(k+1)}\left(\mathbf{x}_{k+1}, \mathbf{x}_{k+1}^{\prime}\right) d \tau
$$

By Minkowski's inequality

$$
\left\|R^{(k)} B_{N, j, k+1} \alpha^{(k+1)}\right\|_{L_{t}^{2} L_{\mathbf{x}, \mathbf{x}^{\prime}}^{2}} \leq \int_{\tau}\left\|R^{(k)} B_{N, j, k+1} U^{(k+1)}(t) f^{(k+1)}\right\|_{L_{t}^{2} L_{\mathbf{x}, \mathbf{x}^{\prime}}^{2}} d \tau
$$

By Lemma 4.2,

$$
\leq \int_{\tau}\left\|R^{(k+1)} f^{(k+1)}\right\|_{L_{\mathbf{x}, \mathbf{x}^{\prime}}^{2}} d \tau
$$

For any $b>\frac{1}{2}$, we write $1=\langle\tau\rangle^{-b}\langle\tau\rangle^{b}$ and apply Cauchy-Schwarz in $\tau$ to obtain

$$
\leq\left\|\langle\tau\rangle^{b} R^{(k+1)} f^{(k+1)}\right\|_{L_{\tau, \mathbf{x}, \mathbf{x}^{\prime}}^{2}}=\left\|R^{(k+1)} \alpha^{(k+1)}\right\|_{X_{b}^{(k+1)}}
$$

Lemma 4.4. For each $\varepsilon>0$, there is a $C_{\varepsilon}$ independent of $M_{k}, j, k$, and $N$ such that

$$
\begin{aligned}
& \left\|R^{(k)} P_{\leqslant M_{k}}^{(k)} B_{N, j, k+1} U^{(k+1)}(t) f^{(k+1)}\right\|_{L_{t}^{2} L_{\mathbf{x}, \mathbf{x}^{\prime}}^{2}} \\
& \quad \leqslant C_{\varepsilon}\|V\|_{L^{1}} \sum_{M_{k+1} \geqslant M_{k}}\left(\frac{M_{k}}{M_{k+1}}\right)^{1-\varepsilon}\left\|R^{(k+1)} P_{\leqslant M_{k+1}}^{(k+1)} f^{(k+1)}\right\|_{L_{\mathbf{x}, \mathbf{x}^{\prime}}^{2}}
\end{aligned}
$$

where the sum on the right is in $M_{k+1}$, over dyads such that $M_{k+1} \geqslant M_{k}$. In particular, if we drop off the projection $P_{\leqslant M_{k+1}}^{(k+1)}$ on the right hand side of the above estimate, carry out the sum and let $M_{k} \rightarrow \infty$, we have Lemma 4 .2 back. This merely gives a fine structure of Lemma 4.2, but not an alternative proof of Lemma 4.2.

Proof. It suffices to take $k=1$ and prove

$$
\begin{aligned}
& \left\|R^{(1)} P_{\leq M_{1}}^{(1)} B_{N, 1,2}\left(R^{(2)}\right)^{-1} U^{(2)}(t) f^{(2)}\right\|_{L_{t}^{2} L_{\mathbf{x}_{1} \mathbf{x}_{1}^{\prime}}^{2}} \\
\leq & C_{\varepsilon}\|V\|_{L^{1}} \sum_{M_{2} \geqslant M_{1}}\left(\frac{M_{1}}{M_{2}}\right)^{1-\varepsilon}\left\|P_{\leq M_{2}}^{(2)} f^{(2)}\right\|_{L_{\mathbf{x}_{2} \mathbf{x}_{2}^{\prime}}^{2}}
\end{aligned}
$$

where the sum is over dyadic $M_{2}$ such that $M_{2} \geqslant M_{1}$. For convenience, we take only "half" of the operator $B_{N, 1,2}$ : For $\alpha^{(2)}\left(t, x_{1}, x_{2}, x_{1}^{\prime}, x_{2}^{\prime}\right)$, define

$$
\left(\tilde{B}_{N, 1,2} \alpha^{(2)}\right)\left(t, x_{1}, x_{1}^{\prime}\right) \stackrel{\text { def }}{=} \int_{x_{2}} V_{N}\left(x_{1}-x_{2}\right) \alpha^{(2)}\left(t, x_{1}, x_{2}, x_{1}^{\prime}, x_{2}\right) d x_{2}
$$

Note that

$$
\begin{aligned}
\left(R^{(1)}\right. & \left.P_{\leq M_{1}}^{(1)} \tilde{B}_{N, 1,2}\left(R^{(2)}\right)^{-1} U^{(2)}(t) f^{(2)}\right) \widehat{\uparrow}\left(\tau, \xi_{1}, \xi_{1}^{\prime}\right) \\
& =\iint_{\xi_{2}, \xi_{2}^{\prime}} \chi_{\leqslant M_{1}}^{(1)} \delta(\cdots) \frac{\widehat{V_{N}}\left(\xi_{2}+\xi_{2}^{\prime}\right)\left|\xi_{1}\right|}{\left|\xi_{1}-\xi_{2}-\xi_{2}^{\prime}\right|\left|\xi_{2}\right|\left|\xi_{2}^{\prime}\right|} \widehat{f^{(2)}}\left(\xi_{1}-\xi_{2}-\xi_{2}^{\prime}, \xi_{2}, \xi_{1}^{\prime}, \xi_{2}^{\prime}\right) d \xi_{2} d \xi_{2}^{\prime}
\end{aligned}
$$


where $\chi$ represents the Littlewood-Paley multiplier on the Fourier side and

$$
\delta(\cdots)=\delta\left(\tau+\left|\xi_{1}-\xi_{2}-\xi_{2}^{\prime}\right|^{2}+\left|\xi_{2}\right|^{2}-\left|\xi_{1}^{\prime}\right|^{2}-\left|\xi_{2}^{\prime}\right|^{2}\right)
$$

Divide this integration into two pieces:

$$
=\iint_{\left|\xi_{2}\right| \leq\left|\xi_{2}^{\prime}\right|}(\cdots) d \xi_{2} d \xi_{2}^{\prime}+\iint_{\left|\xi_{2}^{\prime}\right| \leq\left|\xi_{2}\right|}(\cdots) d \xi_{2} d \xi_{2}^{\prime}
$$

In the first term, decompose the $\xi_{2}^{\prime}$ integration into dyadic intervals, and in the second term, decompose the $\xi_{2}$ integration into dyadic intervals:

$$
\begin{aligned}
= & A+B \\
= & \left(\sum_{M_{2} \geq M_{1}} \iint_{\left|\xi_{2}\right| \leq\left|\xi_{2}^{\prime}\right|} \chi_{M_{2}}^{2^{\prime}}(\cdots) d \xi_{2} d \xi_{2}^{\prime}+\sum_{M_{2} \geq M_{1}} \iint_{\left|\xi_{2}^{\prime}\right| \leq\left|\xi_{2}\right|} \chi_{M_{2}}^{2}(\cdots) d \xi_{2} d \xi_{2}^{\prime}\right) \\
& +\left(\iint_{\left|\xi_{2}\right| \leq\left|\xi_{2}^{\prime}\right|} \chi_{\leq M_{1}}^{2^{\prime}}(\cdots) d \xi_{2} d \xi_{2}^{\prime}+\iint_{\left|\xi_{2}\right| \leq\left|\xi_{2}^{\prime}\right|} \chi_{\leq M_{1}}^{2}(\cdots) d \xi_{2} d \xi_{2}^{\prime}\right) .
\end{aligned}
$$

The $A$ term is the one that needs elaboration. For $B$, we have

$$
B=\iint_{\left|\xi_{2}\right| \leq\left|\xi_{2}^{\prime}\right|} \chi_{\leqslant M_{1}}^{(1)} \chi_{\leqslant M_{1}}^{2} \chi_{\leq M_{1}}^{2^{\prime}}(\cdots) d \xi_{2} d \xi_{2}^{\prime}+\iint_{\left|\xi_{2}\right| \leq\left|\xi_{2}^{\prime}\right|} \chi_{\leqslant M_{1}}^{(1)} \chi_{\leq M_{1}}^{2} \chi_{\leqslant M_{1}}^{2^{\prime}}(\cdots) d \xi_{2} d \xi_{2}^{\prime}
$$

and thus, by Lemma 4.2, we reach

$$
\|B\|_{L_{\tau}^{2} L_{\xi_{1} \xi_{1}^{\prime}}^{2}} \leqslant C\|V\|_{L^{1}}\left\|P_{\leq M_{1}}^{(2)} f^{(2)}\right\|_{L_{\mathbf{x}_{2} x_{2}^{\prime}}^{2}}
$$

which is part of the right hand side of estimate (4.3).

We are now left with the estimate of $A$. Observe that, in the first integration in $A$, we can insert for free the projection $\chi_{\leq 3 M_{2}}^{1} \chi_{\leq M_{1}}^{1^{\prime}} \chi_{\leq M_{2}}^{2}$ onto $\hat{f}^{(2)}$ and in the second integration, we can insert $\chi_{\leq 3 M_{2}}^{1} \chi_{\leq M_{1}}^{1^{\prime}} \chi_{\leq M_{2}}^{2^{\prime}}$ onto $\hat{f}^{(2)}$.

$$
\begin{aligned}
A= & \sum_{M_{2} \geq M_{1}} \iint_{\left|\xi_{2}\right| \leq\left|\xi_{2}^{\prime}\right|} \chi_{\leq 3 M_{2}}\left(\xi_{1}-\xi_{2}-\xi_{2}^{\prime}\right) \chi_{\leq M_{1}}^{1^{\prime}} \chi_{\leq M_{2}}^{2} \chi_{M_{2}}^{2^{\prime}}(\cdots) d \xi_{2} d \xi_{2}^{\prime} \\
& +\sum_{M_{2} \geq M_{1}} \iint_{\left|\xi_{2}\right| \leq\left|\xi_{2}\right|} \chi_{\leq 3 M_{2}}\left(\xi_{1}-\xi_{2}-\xi_{2}^{\prime}\right) \chi_{\leq M_{1}}^{1^{\prime}} \chi_{\leq M_{2}}^{2^{\prime}} \chi_{M_{2}}^{2}(\cdots) d \xi_{2} d \xi_{2}^{\prime}
\end{aligned}
$$

Then for each piece, we proceed as in Klainerman-Machedon [38], performing CauchySchwarz with respect to measures supported on hypersurfaces and applying the $L_{\tau \xi_{1} \xi_{1}^{\prime}}^{2}$ norm to both sides of the resulting inequality 8 In this manner, it suffices to prove the following estimates, uniform in $\tau^{\prime}=\tau-\left|\xi_{1}^{\prime}\right|^{2}$ :

$$
\iint_{\substack{\left|\xi_{2}^{\prime}\right| M_{2},\left|\xi_{2}\right| \leq M_{2}}} \delta(\cdots) \frac{\left|\xi_{1}\right|^{2}}{\left|\xi_{1}-\xi_{2}-\xi_{2}^{\prime}\right|^{2}\left|\xi_{2}\right|^{2}\left|\xi_{2}^{\prime}\right|^{2}} d \xi_{2} d \xi_{2}^{\prime} \leq C_{\varepsilon}\left(\frac{M_{1}}{M_{2}}\right)^{2(1-\varepsilon)},
$$

\footnotetext{
${ }^{8}$ Notice that $\left\|\widehat{V_{N}}\right\|_{L^{\infty}} \leqslant\left\|V_{N}\right\|_{L^{1}}=\|V\|_{L^{1}}$ i.e. $\widehat{V_{N}}$ is a dummy factor.
} 
(recall that $\left|\xi_{1}\right| \lesssim M_{1} \ll M_{2}$ ) and also

$$
\iint_{\substack{\left|\xi_{2}\right| \sim M_{2},\left|\xi_{2}^{\prime}\right| \leq M_{2}}} \delta(\cdots) \frac{\left|\xi_{1}\right|^{2}}{\left|\xi_{1}-\xi_{2}-\xi_{2}^{\prime}\right|^{2}\left|\xi_{2}\right|^{2}\left|\xi_{2}^{\prime}\right|^{2}} d \xi_{2} d \xi_{2}^{\prime} \leq C_{\varepsilon}\left(\frac{M_{1}}{M_{2}}\right)^{2(1-\varepsilon)} .
$$

In both (4.4) and (4.5),

$$
\delta(\cdots)=\delta\left(\tau^{\prime}+\left|\xi_{1}-\xi_{2}-\xi_{2}^{\prime}\right|^{2}+\left|\xi_{2}\right|^{2}-\left|\xi_{2}^{\prime}\right|^{2}\right) .
$$

By rescaling $\xi_{2} \mapsto M_{2} \xi_{2}$ and $\xi_{2}^{\prime} \mapsto M_{2} \xi_{2}^{\prime}$, (4.4) and (4.5) reduce to, respectively, the following. For $\left|\xi_{1}\right| \ll 1$,

$$
\begin{aligned}
& I\left(\tau^{\prime}, \xi_{1}\right) \stackrel{\text { def }}{=} \iint_{\substack{\left|\xi_{2}^{\prime}\right| \sim 1 \\
\left|\xi_{2}\right| \leq 2}} \delta(\cdots) \frac{\left|\xi_{1}\right|^{2}}{\left|\xi_{1}-\xi_{2}-\xi_{2}^{\prime}\right|^{2}\left|\xi_{2}\right|^{2}\left|\xi_{2}^{\prime}\right|^{2}} d \xi_{2} d \xi_{2}^{\prime} \leq C_{\varepsilon}\left|\xi_{1}\right|^{2(1-\varepsilon)}, \\
& I^{\prime}\left(\tau^{\prime}, \xi_{1}\right) \stackrel{\text { def }}{=} \iint_{\substack{\left|\xi_{2}\right| \sim 1,\left|\xi_{2}\right| \leq 2}} \delta(\cdots) \frac{\left|\xi_{1}\right|^{2}}{\left|\xi_{1}-\xi_{2}-\xi_{2}^{\prime}\right|^{2}\left|\xi_{2}\right|^{2}\left|\xi_{2}^{\prime}\right|^{2}} d \xi_{2} d \xi_{2}^{\prime} \leq C_{\varepsilon}\left|\xi_{1}\right|^{2(1-\varepsilon)} .
\end{aligned}
$$

To be precise, the $\xi_{1}$ in estimates (4.6) and (4.7) is $\xi_{1} / M_{2}$ in estimates (4.4) and (4.5). We shall obtain the upper bound $\left|\xi_{1}\right|^{2} \log \left|\xi_{1}\right|^{-1}$ for both (4.6), (4.7).

First, we prove (4.7). Begin by carrying out the $\xi_{2}^{\prime}$ integral to obtain

$$
I^{\prime}\left(\tau^{\prime}, \xi_{1}\right)=\frac{1}{2}\left|\xi_{1}\right|^{2} \int_{\frac{1}{2} \leq\left|\xi_{2}\right| \leq 2} \frac{H^{\prime}\left(\tau^{\prime}, \xi_{1}, \xi_{2}\right)}{\left|\xi_{1}-\xi_{2}\right|\left|\xi_{2}\right|^{2}} d \xi_{2}
$$

where $H^{\prime}\left(\tau^{\prime}, \xi_{1}, \xi_{2}\right)$ is defined as follows. Let $P^{\prime}$ be the truncated plane defined by

$$
P^{\prime}\left(\tau^{\prime}, \xi_{1}, \xi_{2}\right)=\left\{\xi_{2}^{\prime} \in \mathbb{R}^{3}\left|\left(\xi_{2}^{\prime}-\lambda \omega\right) \cdot \omega=0,\right| \xi_{2}^{\prime} \mid \leq 2\right\}
$$

where

Now let

$$
\omega=\frac{\xi_{1}-\xi_{2}}{\left|\xi_{1}-\xi_{2}\right|}, \quad \lambda=\frac{\tau^{\prime}+\left|\xi_{1}-\xi_{2}\right|^{2}+\left|\xi_{2}\right|^{2}}{2\left|\xi_{1}-\xi_{2}\right|}
$$

$$
H^{\prime}\left(\tau^{\prime}, \xi_{1}, \xi_{2}\right)=\int_{\xi_{2}^{\prime} \in P^{\prime}\left(\tau^{\prime}, \xi_{1}, \xi_{2}\right)} \frac{d \sigma\left(\xi_{2}^{\prime}\right)}{\left|\xi_{1}-\xi_{2}-\xi_{2}^{\prime}\right|^{2}\left|\xi_{2}^{\prime}\right|^{2}}
$$

where the integral is computed with respect to the surface measure on $P^{\prime}$.

Since $\left|\xi_{1}-\xi_{2}\right| \sim 1,\left|\xi_{2}\right| \sim 1$, we have the following reduction

$$
I^{\prime}\left(\tau^{\prime}, \xi_{1}\right) \lesssim\left|\xi_{1}\right|^{2} \int_{\frac{1}{2} \leq\left|\xi_{2}\right| \leq 2} H^{\prime}\left(\tau^{\prime}, \xi_{1}, \xi_{2}\right) d \xi_{2}
$$

We now evaluate $H^{\prime}\left(\tau^{\prime}, \xi_{1}, \xi_{2}\right)$. Introduce polar coordinates $(\rho, \theta)$ on the plane $P^{\prime}$ with respect to the "center" $\lambda \omega$, and note that

$$
\begin{aligned}
\left|\xi_{1}-\xi_{2}-\xi_{2}^{\prime}\right|^{2} & =|| \xi_{1}-\xi_{2}\left|\omega-\xi_{2}^{\prime}\right|^{2} \\
& =\left|\left(\left|\xi_{1}-\xi_{2}\right|-\lambda\right) \omega-\left(\xi_{2}^{\prime}-\lambda \omega\right)\right|^{2} \\
& =\left(\left|\xi_{1}-\xi_{2}\right|-\lambda\right)^{2}+\left|\xi_{2}^{\prime}-\lambda \omega\right|^{2} \\
& =\left(\left|\xi_{1}-\xi_{2}\right|-\lambda\right)^{2}+\rho^{2} \\
& =\alpha^{2}+\rho^{2}
\end{aligned}
$$


where

$$
\alpha=\left|\xi_{1}-\xi_{2}\right|-\lambda=\frac{\left|\xi_{1}\right|^{2}-2 \xi_{1} \cdot \xi_{2}-\tau^{\prime}}{2\left|\xi_{1}-\xi_{2}\right|}
$$

Also,

$$
\left|\xi_{2}^{\prime}\right|^{2}=\left|\left(\xi_{2}^{\prime}-\lambda \omega\right)+\lambda \omega\right|^{2}=\left|\xi_{2}^{\prime}-\lambda \omega\right|^{2}+\lambda^{2}=\rho^{2}+\lambda^{2}
$$

Using (4.9) and (4.10) in (4.8),

$$
H^{\prime}\left(\tau^{\prime}, \xi_{1}, \xi_{2}\right)=\int_{0}^{\sqrt{4-\lambda^{2}}} \frac{2 \pi \rho d \rho}{\left(\rho^{2}+\alpha^{2}\right)\left(\rho^{2}+\lambda^{2}\right)}
$$

The restriction to $0 \leq \rho \leq \sqrt{4-\lambda^{2}}$ arises from the fact that the plane $P^{\prime}$ must sit within the ball $\left|\xi_{2}^{\prime}\right| \leq 2$. In particular, $H^{\prime}\left(\tau, \xi_{1}, \xi_{2}\right)=0$ if $|\lambda| \geq 2$ since then the plane $P^{\prime}$ is located entirely outside the ball $\left|\xi_{2}^{\prime}\right| \leq 2$. Since $|\lambda| \leq 2$, we have $|\alpha| \leq 3$ and $\left|\tau^{\prime}\right| \leq 10$.

We consider the three cases: (A) $|\lambda| \leq \frac{1}{4}$ (which implies $|\alpha| \geq \frac{1}{4}$ ), (B) $|\alpha| \leq \frac{1}{4}$ (which implies $|\lambda| \geq \frac{1}{4}$ ), and (C) $|\lambda| \geq \frac{1}{4}$ and $|\alpha| \geq \frac{1}{4}$. Case (C) is the easiest since clearly $\left|H^{\prime}\left(\tau^{\prime}, \xi_{1}, \xi_{2}\right)\right| \leq C$.

Let us consider case (B). Then

$$
H^{\prime}\left(\tau, \xi_{1}, \xi_{2}\right) \lesssim \int_{\rho=0}^{2} \frac{\rho d \rho}{\rho^{2}+\alpha^{2}}=\int_{\nu=0}^{\sqrt{2}} \frac{d \nu}{\nu+\alpha^{2}}=\log \left(1+\frac{\sqrt{2}}{\alpha^{2}}\right)
$$

Substituting back into $I^{\prime}$,

$$
I^{\prime}\left(\tau^{\prime}, \xi_{1}\right) \lesssim\left|\xi_{1}\right|^{2} \int_{\left|\xi_{2}\right| \leq 2} \log \left(1+\frac{\sqrt{2}}{\alpha^{2}}\right) d \xi_{2}
$$

Since $|\alpha| \leq \sqrt{3}$, it follows that 9

$$
\begin{aligned}
\log \left(1+\frac{\sqrt{2}}{\alpha^{2}}\right) & \leq c+|\log | \alpha|| \\
& \leq c+|\log |\left(\left|\xi_{1}\right|^{2}-2 \xi_{1} \cdot \xi_{2}-\tau^{\prime}\right)|| \\
& =c+|\log 2| \xi_{1} \cdot\left(\xi_{2}-\frac{1}{2} \xi_{1}+\frac{\tau^{\prime} \xi_{1}}{2\left|\xi_{1}\right|^{2}}\right) \mid \\
& =c+|\log | \xi_{1} \cdot\left(\xi_{2}-\frac{1}{2} \xi_{1}+\frac{\tau^{\prime} \xi_{1}}{2\left|\xi_{1}\right|^{2}}\right) \mid
\end{aligned}
$$

Hence

$$
I^{\prime}\left(\tau^{\prime}, \xi_{1}\right) \lesssim\left|\xi_{1}\right|^{2}\left(1+\int_{\left|\xi_{2}\right| \leq 2}|\log | \xi_{1} \cdot\left(\xi_{2}-\frac{1}{2} \xi_{1}+\frac{\tau^{\prime} \xi_{1}}{2\left|\xi_{1}\right|^{2}}\right) \mid d \xi_{2}\right)
$$

\footnotetext{
${ }^{9}$ The first step is simply: if $x \geq \delta>0$, then $\log (1+x) \leq \log x+\log \left(1+\frac{1}{\delta}\right)$. The second step uses that $\left|\xi_{1}-\xi_{2}\right| \sim 1$, which follows since $\left|\xi_{1}\right| \ll 1$ and $\left|\xi_{2}\right| \sim 1$.
} 
Denoting by $B(\mu, r)$ the ball of center $\mu$ and radius $r$, the substitution $\xi_{2} \mapsto \xi_{2}+\frac{1}{2} \xi_{1}-\frac{\tau^{\prime} \xi_{1}}{2\left|\xi_{1}\right|^{2}}$ yields, with $\mu=\frac{1}{2} \xi_{1}-\frac{\tau^{\prime} \xi_{1}}{2\left|\xi_{1}\right|^{2}}$,

$$
\begin{aligned}
I^{\prime}\left(\tau^{\prime}, \xi_{1}\right) & \lesssim\left|\xi_{1}\right|^{2}\left(1+\int_{B(\mu, 2)}|\log | \xi_{1} \cdot \xi_{2}|| d \xi_{2}\right) \\
& \lesssim\left|\xi_{1}\right|^{2}\left(\log \left|\xi_{1}\right|^{-1}+\int_{B(\mu, 2)}|\log | \frac{\xi_{1}}{\left|\xi_{1}\right|} \cdot \xi_{2}|| d \xi_{2}\right)
\end{aligned}
$$

By rotating coordinates so that $\frac{\xi_{1}}{\left|\xi_{1}\right|}=(1,0,0)$, and letting $\mu^{\prime}$ denote the corresponding rotation of $\mu$,

$$
I^{\prime}\left(\tau^{\prime}, \xi_{1}\right) \lesssim\left|\xi_{1}\right|^{2}\left(\log \left|\xi_{1}\right|^{-1}+\int_{B\left(\mu^{\prime}, 2\right)}|\log |\left(\xi_{2}\right)_{1} \mid d \xi_{2}\right)
$$

where $\left(\xi_{2}\right)_{1}$ denotes the first coordinate of the vector $\xi_{2}$. Since $\left|\tau^{\prime}\right| \leq 10$, it follows that $\left|\mu^{\prime}\right| \lesssim\left|\xi_{1}\right|^{-1}$ and we finally obtain

$$
I^{\prime}\left(\tau^{\prime}, \xi_{1}\right) \lesssim\left|\xi_{1}\right|^{2} \log \left|\xi_{1}\right|^{-1}
$$

as claimed, completing Case (B).

Case $(\mathrm{A})$ is similar except that we begin with the bound

$$
H^{\prime}\left(\tau^{\prime}, \xi_{1}, \xi_{2}\right) \lesssim \int_{\rho=0}^{2} \frac{2 \pi \rho d \rho}{\rho^{2}+\lambda^{2}}
$$

This completes the proof of (4.7).

Next, we prove (4.6). In the integral defining $I\left(\tau^{\prime}, \xi_{1}\right)$, we have the restriction $\frac{1}{2} \leq\left|\xi_{2}^{\prime}\right| \leq 2$ and $\left|\xi_{2}\right| \leq 2$. Note that if $\frac{1}{4} \leq\left|\xi_{2}\right| \leq 2$, then the argument above that provided the bound for $I^{\prime}\left(\tau^{\prime}, \xi_{1}\right)$ applies. Hence it suffices to restrict to $\left|\xi_{2}\right| \leq \frac{1}{4}$, from which it follows that $\left|\xi_{1}-\xi_{2}-\xi_{2}^{\prime}\right| \sim 1$.

Begin by carrying out the $\xi_{2}^{\prime}$ integral to obtain

$$
I\left(\tau^{\prime}, \xi_{1}\right)=\frac{1}{2}\left|\xi_{1}\right|^{2} \int_{\left|\xi_{2}\right| \leq 2} \frac{H\left(\tau^{\prime}, \xi_{1}, \xi_{2}\right)}{\left|\xi_{1}-\xi_{2}\right|\left|\xi_{2}\right|^{2}} d \xi_{2}
$$

where $H\left(\tau^{\prime}, \xi_{1}, \xi_{2}\right)$ is defined as follows. Let $P$ be the truncated plane defined by

$$
P\left(\tau^{\prime}, \xi_{1}, \xi_{2}\right)=\left\{\xi_{2}^{\prime} \in \mathbb{R}^{3}\left|\left(\xi_{2}^{\prime}-\lambda \omega\right) \cdot \omega=0, \frac{1}{2} \leq\right| \xi_{2}^{\prime} \mid \leq 2\right\}
$$

where

$$
\omega=\frac{\xi_{1}-\xi_{2}}{\left|\xi_{1}-\xi_{2}\right|}, \quad \lambda=\frac{\tau^{\prime}+\left|\xi_{1}-\xi_{2}\right|^{2}+\left|\xi_{2}\right|^{2}}{2\left|\xi_{1}-\xi_{2}\right|}
$$

Now let

$$
H\left(\tau^{\prime}, \xi_{1}, \xi_{2}\right)=\int_{\xi_{2}^{\prime} \in P\left(\tau^{\prime}, \xi_{1}, \xi_{2}\right)} \frac{d \sigma\left(\xi_{2}^{\prime}\right)}{\left|\xi_{1}-\xi_{2}-\xi_{2}^{\prime}\right|^{2}\left|\xi_{2}^{\prime}\right|^{2}}
$$


where the integral is computed with respect to the surface measure on $P$. Since $\left|\xi_{1}-\xi_{2}-\xi_{2}^{\prime}\right| \sim$ 1 and $\left|\xi_{2}^{\prime}\right| \sim 1$, we obtain $H\left(\tau^{\prime}, \xi_{1}, \xi_{2}\right) \leq C$. Substituting into (4.11), we obtain

$$
\begin{aligned}
I\left(\tau^{\prime}, \xi_{1}\right) & \lesssim\left|\xi_{1}\right|^{2} \int_{\left|\xi_{2}\right| \leq \frac{1}{4}} \frac{d \xi_{2}}{\left|\xi_{1}-\xi_{2}\right|\left|\xi_{2}\right|^{2}} \\
& \lesssim\left|\xi_{1}\right|^{2}\left(\int_{\left|\xi_{2}\right| \leq 2\left|\xi_{1}\right|} \frac{d \xi_{2}}{\left|\xi_{1}-\xi_{2}\right|\left|\xi_{2}\right|^{2}}+\int_{2\left|\xi_{1}\right| \leq\left|\xi_{2}\right| \leq \frac{1}{4}} \frac{d \xi_{2}}{\left|\xi_{1}-\xi_{2}\right|\left|\xi_{2}\right|^{2}}\right)
\end{aligned}
$$

In the first integral, we change variables $\xi_{2}=\left|\xi_{1}\right| \eta$, and in the second integral, we use the bound $\left|\xi_{1}-\xi_{2}\right|^{-1} \leq 2\left|\xi_{2}\right|^{-1}$ to obtain

$$
\lesssim\left|\xi_{1}\right|^{2}\left(\int_{|\eta| \leq 2} \frac{d \eta}{\left|\frac{\xi_{1}}{\left|\xi_{1}\right|}-\eta\right||\eta|^{2}}+\int_{2\left|\xi_{1}\right| \leq\left|\xi_{2}\right| \leq \frac{1}{4}} \frac{d \xi_{2}}{\left|\xi_{2}\right|^{3}}\right) \lesssim\left|\xi_{1}\right|^{2} \log \left|\xi_{1}\right|^{-1}
$$

This completes the proof of (4.6).

Lemma 4.5. For each $\varepsilon>0$, there is a $C_{\varepsilon}$ independent of $M_{k}, j, k$, and $N$ such that

$$
\begin{aligned}
& \left\|R^{(k)} P_{\leqslant M_{k}}^{(k)} B_{N, j, k+1} \alpha^{(k+1)}\right\|_{L_{t}^{2} L_{\mathbf{x}, \mathbf{x}^{\prime}}^{2}} \\
& \quad \leqslant C_{\varepsilon} \sum_{M_{k+1} \geqslant M_{k}}\left(\frac{M_{k}}{M_{k+1}}\right)^{1-\varepsilon}\left\|R^{(k+1)} P_{\leqslant M_{k+1}}^{(k+1)} \alpha^{(k+1)}\right\|_{X_{\frac{1}{2}+}^{(k)}} .
\end{aligned}
$$

where the sum on the right is in $M_{k+1}$, over dyads such that $M_{k+1} \geqslant M_{k}$.

Proof. The proof is exactly the same as deducing Lemma 4.3 from Lemma 4.2. We include the proof for completeness. Let

$$
f_{\tau}^{(k+1)}\left(\mathbf{x}_{k+1}, \mathbf{x}_{k+1}^{\prime}\right)=\mathcal{F}_{t \mapsto \tau}\left(U^{(k+1)}(-t) \alpha^{(k+1)}\left(t, \mathbf{x}_{k+1}, \mathbf{x}_{k+1}^{\prime}\right)\right)
$$

where $\mathcal{F}_{t \mapsto \tau}$ denotes the Fourier transform in $t \mapsto \tau$. Then

$$
\alpha^{(k+1)}\left(t, \mathbf{x}_{k+1}, \mathbf{x}_{k+1}^{\prime}\right)=\int_{\tau} e^{i t \tau} U^{(k+1)}(t) f^{(k+1)}\left(\mathbf{x}_{k+1}, \mathbf{x}_{k+1}^{\prime}\right) d \tau
$$

By Minkowski's inequality

$$
\left\|R^{(k)} P_{\leqslant M_{k}}^{(k)} B_{N, j, k+1} \alpha^{(k+1)}\right\|_{L_{t}^{2} L_{\mathbf{x}, \mathbf{x}^{\prime}}^{2}} \leq \int_{\tau}\left\|R^{(k)} P_{\leqslant M_{k}}^{(k)} B_{N, j, k+1} U^{(k+1)}(t) f^{(k+1)}\right\|_{L_{t}^{2} L_{\mathbf{x}, \mathbf{x}^{\prime}}^{2}} d \tau
$$

By Lemma 4.4,

$$
\leq C_{\varepsilon} \sum_{M_{k+1} \geqslant M_{k}}\left(\frac{M_{k}}{M_{k+1}}\right)^{1-\varepsilon} \int_{\tau}\left\|R^{(k+1)} P_{\leqslant M_{k+1}}^{(k+1)} f^{(k+1)}\right\|_{L_{\mathbf{x}, \mathbf{x}^{\prime}}^{2}} d \tau
$$

For any $b>\frac{1}{2}$, we write $1=\langle\tau\rangle^{-b}\langle\tau\rangle^{b}$ and apply Cauchy-Schwarz in $\tau$ to obtain

$$
\begin{aligned}
& \leq C_{\varepsilon} \sum_{M_{k+1} \geqslant M_{k}}\left(\frac{M_{k}}{M_{k+1}}\right)^{1-\varepsilon}\left\|\langle\tau\rangle^{b} R^{(k+1)} P_{\leqslant M_{k+1}}^{(k+1)} f^{(k+1)}\right\|_{L_{\tau, \mathbf{x}, \mathbf{x}^{\prime}}^{2}} \\
& =C_{\varepsilon} \sum_{M_{k+1} \geqslant M_{k}}\left(\frac{M_{k}}{M_{k+1}}\right)^{1-\varepsilon}\left\|R^{(k+1)} P_{\leqslant M_{k+1}}^{(k+1)} \alpha^{(k+1)}\right\|_{X_{\frac{1}{2}+}^{(k)}} .
\end{aligned}
$$




\subsection{A Strichartz estimate.}

Lemma 4.6. Assume $\gamma^{(k)}\left(t, \mathbf{x}_{k} ; \mathbf{x}_{k}^{\prime}\right)$ satisfies the symmetric condition (1.2). Let

$$
\beta^{(k)}\left(t, \mathbf{x}_{k} ; \mathbf{x}_{k}^{\prime}\right)=V\left(x_{i}-x_{j}\right) \gamma^{(k)}\left(t, \mathbf{x}_{k} ; \mathbf{x}_{k}^{\prime}\right)
$$

Then we have the estimates:

$$
\begin{aligned}
\left\|\beta^{(k)}\right\|_{X_{-\frac{1}{2}+}^{(k)}} & \lesssim\|V\|_{L_{x}^{\frac{6}{5}+}}\left\|\left\langle\nabla_{x_{i}}\right\rangle\left\langle\nabla_{x_{j}}\right\rangle \gamma^{(k)}\right\|_{L_{t}^{2} L_{x, x^{\prime}}^{2}}, \\
\left\|\beta^{(k)}\right\|_{X_{-\frac{1}{2}+}^{(k)}} & \lesssim\|V\|_{L_{x}^{3+}}\left\|\gamma^{(k)}\right\|_{L_{t}^{2} L_{x, x^{\prime}}^{2}}, \\
\left\|\beta^{(k)}\right\|_{X_{-\frac{1}{2}+}^{(k)}} & \lesssim\|V\|_{L_{x}^{2+}}\left\|\left\langle\nabla_{x_{i}}\right\rangle^{\frac{1}{2}} \gamma^{(k)}\right\|_{L_{t}^{2} L_{x, x^{\prime}}^{2}} .
\end{aligned}
$$

Proof. It suffices to prove Lemma 4.6 for $k=2$. Since we will need to deal with Fourier transforms in only selected coordinates, we introduce the following notation: $\mathcal{F}_{0}$ denotes Fourier transform in $t, \mathcal{F}_{j}$ denotes Fourier transform in $x_{j}$, and $\mathcal{F}_{j^{\prime}}$ denotes Fourier transform in $x_{j}^{\prime}$. Fourier transforms in multiple coordinates will denoted as combined subscripts - for example, $\mathcal{F}_{01^{\prime}}=\mathcal{F}_{0} \mathcal{F}_{1^{\prime}}$ denotes the Fourier transform in $t$ and $x_{1}^{\prime} 10$

We start by splitting $\gamma^{(2)}$ into the piece where $\left|\xi_{1}\right| \geqslant\left|\xi_{2}\right|$ and the piece where $\left|\xi_{2}\right| \geqslant\left|\xi_{1}\right|$ :

$$
\gamma^{(2)}=\gamma_{\left|\xi_{1}\right| \geqslant\left|\xi_{2}\right|}^{(2)}+\gamma_{\left|\xi_{2}\right| \geqslant\left|\xi_{1}\right|}^{(2)}
$$

The below represents the treatment of

$$
\beta_{\left|\xi_{2}\right| \geqslant\left|\xi_{1}\right|}^{(2)}=V\left(x_{1}-x_{2}\right) \gamma_{\left|\xi_{2}\right| \geqslant\left|\xi_{1}\right|}^{(2)}
$$

since the $\left|\xi_{1}\right| \geqslant\left|\xi_{2}\right|$ case is similar. Let $T$ denote the translation operator

$$
(T f)\left(x_{1}, x_{2}\right)=f\left(x_{1}+x_{2}, x_{2}\right)
$$

Suppressing the $x_{1}^{\prime}, x_{2}^{\prime}$ dependence, we have

$$
\left(\mathcal{F}_{12} T \beta_{\left|\xi_{2}\right| \geqslant\left|\xi_{1}\right|}^{(2)}\right)\left(t, \xi_{1}, \xi_{2}\right)=\left(\mathcal{F}_{12} \beta_{\left|\xi_{2}\right| \geqslant\left|\xi_{1}\right|}^{(2)}\right)\left(t, \xi_{1}, \xi_{2}-\xi_{1}\right)
$$

Also

$$
e^{-2 i t \xi_{1} \cdot \xi_{2}}\left(\mathcal{F}_{12} T \beta_{\left|\xi_{2}\right| \geqslant\left|\xi_{1}\right|}^{(2)}\right)\left(t, \xi_{1}, \xi_{2}\right)=\mathcal{F}_{1}\left[\left(\mathcal{F}_{2} T \beta_{\left|\xi_{2}\right| \geqslant\left|\xi_{1}\right|}^{(2)}\right)\left(t, x_{1}-2 t \xi_{2}, \xi_{2}\right)\right]\left(\xi_{1}\right)
$$

Now

$$
\begin{aligned}
& \left(\mathcal{F}_{012} \beta_{\left|\xi_{2}\right| \geqslant\left|\xi_{1}\right|}^{(2)}\right)\left(\tau-\left|\xi_{2}\right|^{2}+2 \xi_{1} \cdot \xi_{2}, \xi_{1}, \xi_{2}-\xi_{1}\right) \\
& =\left(\mathcal{F}_{012} T \beta_{\left|\xi_{2}\right| \geqslant\left|\xi_{1}\right|}^{(2)}\right)\left(\tau-\left|\xi_{2}\right|^{2}+2 \xi_{1} \cdot \xi_{2}, \xi_{1}, \xi_{2}\right) \quad \text { by (4.16) } \\
& =\mathcal{F}_{0}\left[e^{i t\left|\xi_{2}\right|^{2}} e^{-2 i t \xi_{1} \cdot \xi_{2}}\left(\mathcal{F}_{12} T \beta_{\left|\xi_{2}\right| \geqslant\left|\xi_{1}\right|}^{(2)}\right)\left(t, \xi_{1}, \xi_{2}\right)\right](\tau) \\
& =\mathcal{F}_{0}\left[e^{i t\left|\xi_{2}\right|^{2}} \mathcal{F}_{1}\left[\left(\mathcal{F}_{2} T \beta_{\left|\xi_{2}\right| \geqslant\left|\xi_{1}\right|}^{(2)}\right)\left(t, x_{1}-2 t \xi_{2}, \xi_{2}\right)\right]\left(\xi_{1}\right)\right](\tau) \text { by (4.17) } \\
& =\mathcal{F}_{01}\left[e^{i t\left|\xi_{2}\right|^{2}}\left(\mathcal{F}_{2} T \beta_{\left|\xi_{2}\right| \geqslant\left|\xi_{1}\right|}^{(2)}\right)\left(t, x_{1}-2 t \xi_{2}, \xi_{2}\right)\right]\left(\tau, \xi_{1}\right)
\end{aligned}
$$

\footnotetext{
${ }^{10} \mathrm{We}$ are going to apply the endpoint Strichartz estimate on the non-transformed coordinates. We do not know the origin of such a technique, although it was also used by the first author in [13, Lemma 6].
} 
By changing variables $\xi_{2} \mapsto \xi_{2}-\xi_{1}$ and then changing $\tau \mapsto \tau-\left|\xi_{2}\right|^{2}+2 \xi_{1} \cdot \xi_{2}$, we obtain

$$
\begin{aligned}
& \left\|\beta_{\left|\xi_{2}\right| \geqslant\left|\xi_{1}\right|}^{(2)}\right\|_{X_{-\frac{1}{2}+}^{(2)}} \\
& =\left\|\left(\beta_{\left|\xi_{2}\right| \geqslant\left|\xi_{1}\right|}^{(2)}\right) \uparrow\left(\tau, \xi_{1}, \xi_{2}, \xi_{1}^{\prime}, \xi_{2}^{\prime}\right)\left\langle\tau+\left|\xi_{1}\right|^{2}+\left|\xi_{2}\right|^{2}-\left|\xi_{1}^{\prime}\right|^{2}-\left|\xi_{2}^{\prime}\right|^{2}\right\rangle^{-\frac{1}{2}+}\right\|_{L_{\tau \xi_{1} \xi_{2} \xi_{1}^{\prime} \xi_{2}^{\prime}}^{2}} \\
& =\|\left(\beta_{\left|\xi_{2}\right| \geqslant\left|\xi_{1}\right|}^{(2)}\right) \uparrow\left(\tau-\left|\xi_{2}\right|^{2}+2 \xi_{1} \cdot \xi_{2}, \xi_{1}, \xi_{2}-\xi_{1}, \xi_{1}^{\prime}, \xi_{2}^{\prime}\right) \\
& \quad \times\left\langle\tau+2\left|\xi_{1}\right|^{2}-\left|\xi_{1}^{\prime}\right|^{2}-\left|\xi_{2}^{\prime}\right|^{2}\right\rangle^{-\frac{1}{2}+} \|_{L_{\tau \xi_{1} \xi_{2} \xi_{1}^{\prime} \xi_{2}^{\prime}}}
\end{aligned}
$$

Applying the the dual Strichartz (see (4.20) below), the above is bounded by

$$
\lesssim\left\|\mathcal{F}_{01}^{-1}\left[\left(\mathcal{F}_{012} \beta_{\left|\xi_{2}\right| \geqslant\left|\xi_{1}\right|}^{(2)}\right)\left(\tau-\left|\xi_{2}\right|^{2}+2 \xi_{1} \cdot \xi_{2}, \xi_{1}, \xi_{2}-\xi_{1}\right)\right]\left(t, x_{1}\right)\right\|_{L_{\xi_{2}}^{2} L_{t}^{2} L_{x_{1}}^{\frac{6}{5}+} L_{x_{1}^{\prime} x_{2}^{\prime}}^{2}}
$$

Utilizing (4.18), the above is equal to

$$
=\left\|\left(\mathcal{F}_{2} T \beta_{\left|\xi_{2}\right| \geqslant\left|\xi_{1}\right|}^{(2)}\right)\left(t, x_{1}-2 t \xi_{2}, \xi_{2}\right)\right\|_{L_{t}^{2} L_{\xi_{2}}^{2} L_{x_{1}}^{\frac{6}{5}+} L_{x_{1}^{\prime} x_{2}^{\prime}}^{2}}
$$

Change variable in $x_{1} \mapsto x_{1}+2 t \xi_{2}$ to obtain

$$
=\left\|\left(\mathcal{F}_{2} T \beta_{\left|\xi_{2}\right| \geqslant\left|\xi_{1}\right|}^{(2)}\right)\left(t, x_{1}, \xi_{2}\right)\right\|_{L_{t}^{2} L_{\xi_{2}}^{2} L_{x_{1}}^{\frac{6}{5}+} L_{x_{1}^{\prime} x_{2}^{\prime 2}}^{2}}
$$

Now note that from (4.12), we have

$$
\left(\mathcal{F}_{2} T \beta_{\left|\xi_{2}\right| \geqslant\left|\xi_{1}\right|}^{(2)}\right)\left(t, x_{1}, \xi_{2}\right)=V\left(x_{1}\right)\left(\mathcal{F}_{2} T \gamma_{\left|\xi_{2}\right| \geqslant\left|\xi_{1}\right|}^{(2)}\right)\left(t, x_{1}, \xi_{2}\right)
$$

It follows that

$$
\begin{aligned}
& \left\|\left(\mathcal{F}_{2} T \beta_{\left|\xi_{2}\right| \geqslant\left|\xi_{1}\right|}^{(2)}\right)\left(t, x_{1}, \xi_{2}\right)\right\|_{L_{t}^{2} L_{\xi_{2}}^{2} L_{x_{1}}^{\frac{6}{5}+} L_{x_{1}^{\prime} x_{2}^{\prime}}^{2}} \\
& \quad=\left\|V\left(x_{1}\right)\left(\left\|\left(\mathcal{F}_{2} T \gamma_{\left|\xi_{2}\right| \geqslant\left|\xi_{1}\right|}^{(2)}\right)\left(t, x_{1}, \xi_{2}\right)\right\|_{L_{x_{1}^{\prime} x_{2}^{\prime}}^{2}}\right)\right\|_{L_{t}^{2} L_{\xi_{2}}^{2} L_{x_{1}}^{\frac{6}{5}+}} \\
& \quad \leq\|V\|_{L^{\frac{6}{5}+}}\left\|\left(\mathcal{F}_{2} T \gamma_{\left|\xi_{2}\right| \geqslant\left|\xi_{1}\right|}^{(2)}\right)\left(t, x_{1}, \xi_{2}\right)\right\|_{L_{t}^{2} L_{\xi_{2}}^{2} L_{x_{1}}^{\infty} L_{x_{1}^{\prime} x_{2}^{\prime}}^{2}} \\
& \leq\|V\|_{L^{\frac{6}{5}+}}\left\|\left(\mathcal{F}_{2} T \gamma_{\left|\xi_{2}\right| \geqslant\left|\xi_{1}\right|}^{(2)}\right)\left(t, x_{1}, \xi_{2}\right)\right\|_{L_{t}^{2} L_{\xi_{2} x_{1}^{\prime} x_{2}^{\prime}}^{2} L_{x_{1}}^{\infty}}
\end{aligned}
$$

Apply Sobolev in $x_{1}$ :

$$
\lesssim\|V\|_{L^{\frac{6}{5}}}\left\|\left\langle\nabla_{x_{1}}\right\rangle^{2}\left(\mathcal{F}_{2} T \gamma_{\left|\xi_{2}\right| \geqslant\left|\xi_{1}\right|}^{(2)}\right)\left(t, x_{1}, \xi_{2}\right)\right\|_{L_{t}^{2} L_{\xi_{2} x_{1}^{\prime} x_{2}^{\prime}}^{2} L_{x_{1}}^{2}}
$$

Move the $d \xi_{2} d x_{1}^{\prime} d x_{2}^{\prime}$ integration to the inside and apply Plancherel in $\xi_{2} \mapsto x_{2}$ to obtain

$$
\begin{aligned}
& =\|V\|_{L^{\frac{6}{5}}+}\left\|\left\langle\nabla_{x_{1}}\right\rangle^{2} T \gamma_{\left|\xi_{2}\right| \geqslant\left|\xi_{1}\right|}^{(2)}\right\|_{L_{t}^{2} L_{\mathbf{x}, \mathbf{x}^{\prime}}^{2}} \\
& =\|V\|_{L^{\frac{6}{5}}+}\left\|\left\langle\nabla_{x_{1}}\right\rangle^{2} \gamma_{\left|\xi_{2}\right| \geqslant\left|\xi_{1}\right|}^{(2)}\right\|_{L_{t}^{2} L_{\mathbf{x}, \mathbf{x}^{\prime}}^{2}} \text {. }
\end{aligned}
$$


Recall that the $\xi_{2}$ frequency dominates in $\gamma_{\left|\xi_{2}\right| \geqslant\left|\xi_{1}\right|}^{(2)}$, and thus this is bounded above by

$$
\begin{aligned}
& \lesssim\|V\|_{L^{\frac{6}{5}}+}\left\|\left\langle\nabla_{x_{1}}\right\rangle\left\langle\nabla_{x_{2}}\right\rangle \gamma_{\left|\xi_{2}\right| \geqslant\left|\xi_{1}\right|}^{(2)}\left(t, \mathbf{x}_{2}, \mathbf{x}_{2}^{\prime}\right)\right\|_{L_{t}^{2} L_{\mathbf{x}, \mathbf{x}^{\prime}}^{2}} \\
& \lesssim\|V\|_{L^{\frac{6}{5}}+}\left\|\left\langle\nabla_{x_{1}}\right\rangle\left\langle\nabla_{x_{2}}\right\rangle \gamma^{(2)}\left(t, \mathbf{x}_{2}, \mathbf{x}_{2}^{\prime}\right)\right\|_{L_{t}^{2} L_{\mathbf{x}, \mathbf{x}^{\prime}}^{2}} .
\end{aligned}
$$

This proves estimate (4.13). Using Hölder exponents $\left(3+, 2, \frac{6}{5}+\right)$ and $\left(2+, 3, \frac{6}{5}+\right)$ in (4.19) yields estimates (4.14) and (4.15). Their proofs are easier in the sense that there is no need to split $\gamma^{(2)}$.

It remains to prove the following dual Strichartz estimate (here $\sigma^{(2)}\left(t, x_{1}, x_{1}^{\prime}, x_{2}^{\prime}\right)$, note that the $x_{2}$ coordinate is missing):

$$
\left\|\left\langle\tau+2\left|\xi_{1}\right|^{2}-\left|\xi_{1}^{\prime}\right|^{2}-\left|\xi_{2}^{\prime}\right|^{2}\right\rangle^{-\frac{1}{2}+} \hat{\sigma}^{(2)}\left(\tau, \xi_{1}, \xi_{1}^{\prime}, \xi_{2}^{\prime}\right)\right\|_{L_{\tau}^{2} L_{\xi_{1} \xi_{1}^{\prime} \xi_{2}^{\prime}}^{2}} \lesssim\left\|\sigma^{(2)}\right\|_{L_{t}^{2} L_{x_{1}}^{\frac{6}{5}+} L_{x_{1}^{\prime} x_{2}^{\prime}}^{2}}
$$

The estimate (4.20) is dual to the equivalent estimate

$$
\left\|\sigma^{(2)}\right\|_{L_{t}^{2} L_{x_{1}}^{6-} L_{x_{1}^{\prime} x_{2}^{\prime}}^{2}} \lesssim\left\|\left\langle\tau+2\left|\xi_{1}\right|^{2}-\left|\xi_{1}^{\prime}\right|^{2}-\left|\xi_{2}^{\prime}\right|^{2}\right\rangle^{\frac{1}{2}-} \hat{\sigma}^{(2)}\left(\tau, \xi_{1}, \xi_{1}^{\prime}, \xi_{2}^{\prime}\right)\right\|_{L_{\tau}^{2} L_{\xi_{1} \xi_{1}^{\prime} \xi_{2}^{\prime}}^{2}}
$$

To prove (4.21), we prove

$$
\left\|\sigma^{(2)}\right\|_{L_{t}^{2} L_{x_{1}}^{6} L_{x_{1}^{\prime} x_{2}^{\prime}}^{2}} \lesssim\left\|\left\langle\tau+2\left|\xi_{1}\right|^{2}-\left|\xi_{1}^{\prime}\right|^{2}-\left|\xi_{2}^{\prime}\right|^{2}\right\rangle^{\frac{1}{2}+} \hat{\sigma}^{(2)}\left(\tau, \xi_{1}, \xi_{1}^{\prime}, \xi_{2}^{\prime}\right)\right\|_{L_{\tau}^{2} L_{\xi_{1} \xi_{1}^{\prime} \xi_{2}^{\prime}}}
$$

The estimate (4.21) follows from the interpolation of (4.22) and the trivial equality

$$
\left\|\sigma^{(2)}\right\|_{L_{t}^{2} L_{x_{1}}^{2} L_{x_{1}^{\prime} x_{2}^{\prime}}^{2}}=\left\|\left\langle\tau+2\left|\xi_{1}\right|^{2}-\left|\xi_{1}^{\prime}\right|^{2}-\left|\xi_{2}^{\prime}\right|^{2}\right\rangle^{0} \hat{\sigma}^{(2)}\left(\tau, \xi_{1}, \xi_{1}^{\prime}, \xi_{2}^{\prime}\right)\right\|_{L_{\tau}^{2} L_{\xi_{1} \xi_{1}^{\prime} \xi_{2}^{\prime}}}
$$

Thus proving (4.20) is reduced to proving (4.22), which we do now. Let

$$
\phi_{\tau}\left(x_{1}, x_{1}^{\prime}, x_{2}\right) \stackrel{\text { def }}{=} \mathcal{F}_{0}\left[U^{1}(-2 t) U^{1^{\prime}}(-t) U^{2^{\prime}}(-t) \sigma^{(2)}\left(t, x_{1}, x_{1}^{\prime}, x_{2}^{\prime}\right)\right](\tau)
$$

Then note $\phi_{\tau}$ is independent of $t$ and

$$
\sigma^{(2)}\left(t, x_{1}, x_{1}^{\prime}, x_{2}^{\prime}\right)=\int e^{i t \tau} U^{1}(2 t) U^{1^{\prime}}(t) U^{2^{\prime}}(t) \phi_{\tau}\left(x_{1}, x_{1}^{\prime}, x_{2}^{\prime}\right) d \tau
$$

Thus

$$
\begin{aligned}
\left\|\sigma^{(2)}\right\|_{L_{t}^{2} L_{x_{1}}^{6} L_{x_{1}^{\prime} x_{2}^{\prime}}^{2}} & \lesssim \int_{\tau}\left\|U^{1^{\prime}}(t) U^{2^{\prime}}(t) U^{1}(2 t) \phi_{\tau}\left(x_{1}, x_{1}^{\prime}, x_{2}^{\prime}\right)\right\|_{L_{t}^{2} L_{x_{1}}^{6} L_{x_{1}^{\prime} x_{2}^{\prime}}^{2}} d \tau \\
& \lesssim \int_{\tau}\left\|U^{1}(2 t) \phi_{\tau}\left(x_{1}, x_{1}^{\prime}, x_{2}^{\prime}\right)\right\|_{L_{t}^{2} L_{x_{1}}^{6} L_{x_{1}^{\prime} x_{2}^{\prime}}^{2}} d \tau \\
& \lesssim \int_{\tau}\left\|U^{1}(2 t) \phi_{\tau}\left(x_{1}, x_{1}^{\prime}, x_{2}^{\prime}\right)\right\|_{L_{x_{1}^{\prime} x_{2}^{\prime}}^{2} L_{t}^{2} L_{x_{1}}^{6}} d \tau
\end{aligned}
$$

Now apply Keel-Tao [34] endpoint Strichartz estimate to obtain

$$
\begin{aligned}
& \lesssim \int_{\tau}\left\|\phi_{\tau}\left(x_{1}, x_{1}^{\prime}, x_{2}^{\prime}\right)\right\|_{L_{x_{1}^{\prime} x_{2}^{\prime}}^{2} L_{x_{1}}^{2}} d \tau \\
& \lesssim\left\|\langle\tau\rangle^{\frac{1}{2}+} \phi_{\tau}\left(x_{1}, x_{1}^{\prime}, x_{2}^{\prime}\right)\right\|_{L_{\tau}^{2} L_{x_{1} x_{1}^{\prime} x_{2}^{\prime}}^{2}}
\end{aligned}
$$

It follows from (4.23) that

$$
=\left\|\left\langle\tau+2\left|\xi_{1}\right|^{2}-\left|\xi_{1}^{\prime}\right|^{2}-\left|\xi_{2}^{\prime}\right|^{2}\right\rangle^{\frac{1}{2}+} \hat{\sigma}^{(2)}\left(\tau, \xi_{1}, \xi_{1}^{\prime}, \xi_{2}^{\prime}\right)\right\|_{L_{\tau \xi_{1} \xi_{1}^{\prime} \xi_{2}^{\prime}}}
$$


which completes the proof of (4.22).

Corollary 4.1. Let

$$
\beta^{(k)}\left(t, \mathbf{x}_{k}, \mathbf{x}_{k}^{\prime}\right)=N^{3 \beta-1} V\left(N^{\beta}\left(x_{i}-x_{j}\right)\right) \gamma^{(k)}\left(t, \mathbf{x}_{k}, \mathbf{x}_{k}^{\prime}\right)
$$

Then for $N \geq 1$, we have

$$
\left\|\left|\nabla_{x_{i}}\right|\left|\nabla_{x_{j}}\right| \beta^{(k)}\right\|_{X_{-\frac{1}{2}+}^{(k)}} \lesssim N^{\frac{5}{2} \beta-1}\left\|\left\langle\nabla_{x_{i}}\right\rangle\left\langle\nabla_{x_{j}}\right\rangle \gamma^{(k)}\right\|_{L_{t}^{2} L_{\mathbf{x x}^{\prime}}}
$$

and

$$
\left\|\beta^{(k)}\right\|_{X_{-\frac{1}{2}+}^{(k)}} \lesssim N^{\frac{1}{2} \beta-1}\left\|\left\langle\nabla_{x_{i}}\right\rangle\left\langle\nabla_{x_{j}}\right\rangle \gamma^{(k)}\right\|_{L_{t}^{2} L_{\mathbf{x x}^{\prime}}^{2}}
$$

Consequently, $\left(R_{\leq M}^{(k)}=P_{\leq M}^{(k)} R^{(k)}\right)$

$$
\left\|R_{\leq M}^{(k)} \beta^{(k)}\right\|_{X_{-\frac{1}{2}+}^{(k)}} \lesssim N^{\frac{1}{2} \beta-1} \min \left(M^{2}, N^{2 \beta}\right)\left\|S^{(k)} \gamma^{(k)}\right\|_{L_{t}^{2} L_{\mathbf{x} \mathbf{x}^{\prime}}^{2}}
$$

Proof. Estimate (4.24) follows by applying either (4.13), (4.14), or (4.15) according to whether two derivatives, no derivatives, or one derivative, respectively, lands on $N^{3 \beta-1} V\left(N^{\beta}\left(x_{i}-\right.\right.$ $\left.\left.x_{j}\right)\right)$.

Estimate (4.25) follows by applying (4.13).

Finally, (4.26) follows from (4.24) and (4.25), as follows. Let

$$
Q=\prod_{\substack{1 \leq \ell \leq k \\ \ell \neq i, j}}\left|\nabla_{x_{\ell}}\right|
$$

Then

$$
\left\|R_{\leq M}^{(k)} \beta^{(k)}\right\|_{X_{-\frac{1}{2}+}^{(k)}} \leq M^{2}\left\|Q \beta^{(k)}\right\|_{X_{-\frac{1}{2}+}^{(k)}}
$$

The $Q$ operator passes directly onto $\gamma^{(k)}$, and one applies (4.25) to obtain

$$
\left\|R_{\leq M}^{(k)} \beta^{(k)}\right\|_{X_{-\frac{1}{2}+}^{(k)}} \lesssim N^{\frac{1}{2} \beta-1} M^{2}\left\|S^{(k)} \gamma^{(k)}\right\|_{L_{t}^{2} L_{\mathbf{x x} \mathbf{x}^{\prime}}}
$$

On the other hand,

$$
\left\|R_{\leq M}^{(k)} \beta^{(k)}\right\|_{X_{-\frac{1}{2}+}^{(k)}} \leq\left\|Q\left|\nabla_{x_{i}}\right|\left|\nabla_{x_{j}}\right| \beta^{(k)}\right\|_{X_{-\frac{1}{2}+}^{(k)}}
$$

The $Q$ operator passes directly on $\gamma^{(k)}$, and one applies (4.24) to obtain

$$
\left\|R_{\leq M}^{(k)} \beta^{(k)}\right\|_{X_{-\frac{1}{2}+}^{(k)}} \lesssim N^{\frac{5}{2} \beta-1}\left\|S^{(k)} \gamma^{(k)}\right\|_{L_{t}^{2} L_{\mathbf{x x}^{\prime}}^{2}}
$$

Combining (4.27) and (4.28), we obtain (4.26). 


\section{Appendix A. The topology on the Density matrices}

In this appendix, we define a topology $\tau_{\text {prod }}$ on the density matrices as was previously done in [20, 21, 22, 23, 24, 25, 36, 9, 14, 15, 16].

Denote the spaces of compact operators and trace class operators on $L^{2}\left(\mathbb{R}^{3 k}\right)$ as $\mathcal{K}_{k}$ and $\mathcal{L}_{k}^{1}$, respectively. Then $\left(\mathcal{K}_{k}\right)^{\prime}=\mathcal{L}_{k}^{1}$. By the fact that $\mathcal{K}_{k}$ is separable, we select a dense countable subset $\left\{J_{i}^{(k)}\right\}_{i \geqslant 1} \subset \mathcal{K}_{k}$ in the unit ball of $\mathcal{K}_{k}$ (so $\left\|J_{i}^{(k)}\right\|_{\text {op }} \leqslant 1$ where $\|\cdot\|_{\text {op }}$ is the operator norm). For $\gamma^{(k)}, \tilde{\gamma}^{(k)} \in \mathcal{L}_{k}^{1}$, we then define a metric $d_{k}$ on $\mathcal{L}_{k}^{1}$ by

$$
d_{k}\left(\gamma^{(k)}, \tilde{\gamma}^{(k)}\right)=\sum_{i=1}^{\infty} 2^{-i}\left|\operatorname{Tr} J_{i}^{(k)}\left(\gamma^{(k)}-\tilde{\gamma}^{(k)}\right)\right| .
$$

A uniformly bounded sequence $\gamma_{N}^{(k)} \in \mathcal{L}_{k}^{1}$ converges to $\gamma^{(k)} \in \mathcal{L}_{k}^{1}$ with respect to the weak* topology if and only if

$$
\lim _{N} d_{k}\left(\gamma_{N}^{(k)}, \gamma^{(k)}\right)=0
$$

For fixed $T>0$, let $C\left([0, T], \mathcal{L}_{k}^{1}\right)$ be the space of functions of $t \in[0, T]$ with values in $\mathcal{L}_{k}^{1}$ which are continuous with respect to the metric $d_{k}$. On $C\left([0, T], \mathcal{L}_{k}^{1}\right)$, we define the metric

$$
\hat{d}_{k}\left(\gamma^{(k)}(\cdot), \tilde{\gamma}^{(k)}(\cdot)\right)=\sup _{t \in[0, T]} d_{k}\left(\gamma^{(k)}(t), \tilde{\gamma}^{(k)}(t)\right)
$$

We can then define a topology $\tau_{\text {prod }}$ on the space $\oplus_{k \geqslant 1} C\left([0, T], \mathcal{L}_{k}^{1}\right)$ by the product of topologies generated by the metrics $\hat{d}_{k}$ on $C\left([0, T], \mathcal{L}_{k}^{1}\right)$.

\section{Appendix B. Proof of estimates (2.8) And (2.10)}

Proof of Estimate (2.8). Utilizing Lemma 2.1 and estimate (3.3) to the free part of $\gamma_{N}^{(2)}$, we obtain

$$
\begin{aligned}
& \left\|R^{(k-1)} B_{N, 1, k} \mathrm{FP}^{\left(k, l_{c}\right)}\right\|_{L_{T}^{1} L_{\mathbf{x}, \mathbf{x}^{\prime}}^{2}} \\
\leqslant & C T^{\frac{1}{2}}\left\|R^{(k)} \gamma_{N, 0}^{(k)}\right\|_{L_{\mathbf{x}, \mathbf{x}^{\prime}}^{2}} \\
& +\sum_{j=1}^{l_{c}} \sum_{m}\left\|R^{(k-1)} B_{N, 1, k} \int_{D} J_{N}^{(k, j)}\left(\underline{t}_{k+j}, \mu_{m}\right)\left(U^{(k+j)}\left(t_{k+j}\right) \gamma_{N, 0}^{(k+j)}\right) d \underline{t}_{k+j}\right\|_{L_{T}^{1} L_{\mathbf{x}, \mathbf{x}^{\prime}}^{2}} \\
\leqslant & C T^{\frac{1}{2}}\left\|R^{(k)} \gamma_{N, 0}^{(k)}\right\|_{L_{\mathbf{x}, \mathbf{x}^{\prime}}^{2}} \\
& +\sum_{j=1}^{l_{c}} \sum_{m}\left(C T^{\frac{1}{2}}\right)^{j} \|_{R^{(k+j-1)} B_{N, \mu_{m}(k+j), k+j} U^{(k+j)}\left(t_{k+j}\right) \gamma_{N, 0}^{(k+j)} \|_{L_{T}^{1} L_{\mathbf{x}, \mathbf{x}^{\prime}}^{2}}} \\
& C T^{\frac{1}{2}}\left\|R^{(k)} \gamma_{N, 0}^{(k)}\right\|_{L_{\mathbf{x}, \mathbf{x}^{\prime}}^{2}}+\sum_{j=1}^{\infty} 4^{j-1}\left(C T^{\frac{1}{2}}\right)^{j+1}\left\|R^{(k+j)} \gamma_{N, 0}^{(k+j)}\right\|_{L_{\mathbf{x}, \mathbf{x}^{\prime}}^{2}}
\end{aligned}
$$


Use condition (2.5), it becomes

$$
\begin{aligned}
& \leqslant C T^{\frac{1}{2}} C_{0}^{k}+\sum_{j=1}^{\infty} 4^{j-1}\left(C T^{\frac{1}{2}}\right)^{j+1} C_{0}^{k+j} \\
& \leqslant C_{0}^{k}\left(C T^{\frac{1}{2}}+\sum_{j=1}^{\infty} 4^{j-1}\left(C T^{\frac{1}{2}}\right)^{j+1} C_{0}^{j}\right) .
\end{aligned}
$$

We can choose a $T$ independent of $k, l_{c}$ and $N$ such that the series in the above estimate converges. We then have

$$
\begin{aligned}
\left\|R^{(k-1)} B_{N, 1, k} \mathrm{FP}^{\left(k, l_{c}\right)}\right\|_{L_{T}^{1} L_{\mathbf{x}, \mathbf{x}^{\prime}}^{2}} & \leqslant C_{0}^{k}\left(C T^{\frac{1}{2}}+C\right) \\
& \leqslant C^{k-1}
\end{aligned}
$$

for some $C$ larger than $C_{0}$. Whence, we have shown estimate (2.8).

Proof of Estimate (2.10). We proceed like the proof of estimate (2.8) and end up with

$$
\begin{aligned}
& \left\|R^{(k-1)} B_{N, 1, k} I P^{\left(k, l_{c}\right)}\right\|_{L_{T}^{1} L_{\mathbf{x}, \mathbf{x}^{\prime}}^{2}} \\
\leqslant & \sum_{m}\left\|R^{(k-1)} B_{N, 1, k} \int_{D} J_{N}^{\left(k, l_{c}+1\right)}\left(\underline{t}_{k+l_{c}+1}, \mu_{m}\right)\left(\gamma_{N}^{\left(k+l_{c}+1\right)}\left(t_{k+l_{c}+1}\right)\right) d \underline{t}_{k+l_{c}+1}\right\|_{L_{T}^{1} L_{\mathbf{x}, \mathbf{x}^{\prime}}^{2}} \\
\leqslant & \sum_{m}\left(C T^{\frac{1}{2}}\right)^{l_{c}}\left\|R^{\left(k+l_{c}\right)} B_{N, \mu_{m}\left(k+l_{c}+1\right), k+l_{c}+1} \gamma_{N}^{\left(k+l_{c}+1\right)}\left(t_{k+l_{c}+1}\right)\right\|_{L_{T}^{1} L_{\mathbf{x}, \mathbf{x}^{\prime}}^{2}} .
\end{aligned}
$$

We then investigate

$$
\left\|R^{\left(k+l_{c}\right)} B_{N, \mu_{m}\left(k+l_{c}+1\right), k+l_{c}+1} \gamma_{N}^{\left(k+l_{c}+1\right)}\left(t_{k+l_{c}+1}\right)\right\|_{L_{T}^{1} L_{\mathbf{x}, \mathbf{x}^{\prime}}^{2}} .
$$

Set $\mu_{m}\left(k+l_{c}+1\right)=1$ for simplicity and look at $\tilde{B}_{N, 1, k+l_{c}+1}$, we have

$$
\begin{aligned}
& R^{\left(k+l_{c}\right)} \tilde{B}_{N, 1, k+l_{c}+1} \gamma_{N}^{\left(k+l_{c}+1\right)}(t) \\
= & R^{\left(k+l_{c}\right)} \int V_{N}\left(x_{1}-x_{k+l_{c}+1}\right) \gamma_{N}^{\left(k+l_{c}+1\right)}\left(t, \mathbf{x}_{k+l_{c}}, x_{k+l_{c}+1} ; \mathbf{x}_{k+l_{c}}^{\prime}, x_{k+l_{c}+1}\right) d x_{k+l_{c}+1} \\
= & I+I I
\end{aligned}
$$

with $I$ and $I I$ given by the product rule:

$$
\begin{aligned}
& I=\int V_{N}^{\prime}\left(x_{1}-x_{k+l_{c}+1}\right)\left(\frac{R^{\left(k+l_{c}\right)}}{\left|\nabla_{x_{1}}\right|}\right) \gamma_{N}^{\left(k+l_{c}+1\right)}\left(t, \mathbf{x}_{k+l_{c}}, x_{k+l_{c}+1} ; \mathbf{x}_{k+l_{c}}^{\prime}, x_{k+l_{c}+1}\right) d x_{k+l_{c}+1}, \\
& I I=\int V_{N}\left(x_{1}-x_{k+l_{c}+1}\right)\left(R^{\left(k+l_{c}\right)} \gamma_{N}^{\left(k+l_{c}+1\right)}\right)\left(t, \mathbf{x}_{k+l_{c}}, x_{k+l_{c}+1} ; \mathbf{x}_{k+l_{c}}^{\prime}, x_{k+l_{c}+1}\right) d x_{k+l_{c}+1},
\end{aligned}
$$

where we wrote

$$
\frac{R^{\left(k+l_{c}\right)}}{\left|\nabla_{x_{1}}\right|}=\left(\prod_{j=2}^{k+l_{c}}\left|\nabla_{x_{j}}\right|\right)\left(\prod_{j=1}^{k+l_{c}}\left|\nabla_{x_{j}^{\prime}}\right|\right) .
$$


Then

$$
\begin{aligned}
& \int\left|R^{\left(k+l_{c}\right)} \tilde{B}_{N, 1, k+l_{c}+1} \gamma_{N}^{\left(k+l_{c}+1\right)}(t)\right|^{2} d \mathbf{x}_{k+l_{c}} d \mathbf{x}_{k+l_{c}}^{\prime} \\
= & \int|I+I I|^{2} d \mathbf{x}_{k+l_{c}} d \mathbf{x}_{k+l_{c}}^{\prime} \\
\leqslant & C \int|I|^{2} d \mathbf{x}_{k+l_{c}} d \mathbf{x}_{k+l_{c}}^{\prime}+C \int|I I|^{2} d \mathbf{x}_{k+l_{c}} d \mathbf{x}_{k+l_{c}}^{\prime} .
\end{aligned}
$$

To estimate the first term, we first Cauchy-Schwarz $d x_{k+l_{c}+1}$,

$$
\begin{aligned}
& \int|I|^{2} d \mathbf{x}_{k+l_{c}} d \mathbf{x}_{k+l_{c}}^{\prime} \\
\leqslant & \int d \mathbf{x}_{k+l_{c}} d \mathbf{x}_{k+l_{c}}^{\prime}\left(\int\left|V_{N}^{\prime}\left(x_{1}-x_{k+l_{c}+1}\right)\right|^{2} d x_{k+l_{c}+1}\right) \\
& \times\left(\int\left|\left(\frac{R^{\left(k+l_{c}\right)}}{\left|\nabla_{x_{1}}\right|}\right) \gamma_{N}^{\left(k+l_{c}+1\right)}\left(t, \mathbf{x}_{k+l_{c}}, x_{k+l_{c}+1} ; \mathbf{x}_{k+l_{c}}^{\prime}, x_{k+l_{c}+1}\right)\right|^{2} d x_{k+l_{c}+1}\right) \\
\leqslant & N^{5 \beta}\left\|V^{\prime}\right\|_{L^{2}}^{2} \int d \mathbf{x}_{k+l_{c}} d \mathbf{x}_{k+l_{c}}^{\prime} \\
& \times\left(\int\left|S^{\left(k+l_{c}\right)} \gamma_{N}^{\left(k+l_{c}+1\right)}\left(t, \mathbf{x}_{k+l_{c}}, x_{k+l_{c}+1} ; \mathbf{x}_{k+l_{c}}^{\prime}, x_{k+l_{c}+1}\right)\right|^{2} d x_{k+l_{c}+1}\right)
\end{aligned}
$$

where $V \in W^{2, \frac{6}{5}+}$ implies that $V \in H^{1}$ by Sobolev. A trace theorem then gives

$$
\begin{aligned}
\leqslant & C N^{5 \beta}\left\|V^{\prime}\right\|_{L^{2}}^{2} \int d \mathbf{x}_{k+l_{c}} d \mathbf{x}_{k+l_{c}}^{\prime} \\
& \times\left(\int\left|S^{\left(k+l_{c}+1\right)} \gamma_{N}^{\left(k+l_{c}+1\right)}\left(t, \mathbf{x}_{k+l_{c}}, x_{k+l_{c}+1} ; \mathbf{x}_{k+l_{c}}^{\prime}, x_{k+l_{c}+1}^{\prime}\right)\right|^{2} d x_{k+l_{c}+1} d x_{k+l_{c}+1}^{\prime}\right) \\
= & C N^{5 \beta}\left\|V^{\prime}\right\|_{L^{2}}^{2}\left\|S^{\left(k+l_{c}+1\right)} \gamma_{N}^{\left(k+l_{c}+1\right)}\right\|_{L_{T}^{\infty} L_{\mathbf{x}, \mathbf{x}^{\prime}}^{2}}^{2} .
\end{aligned}
$$

Estimate the second term in the same manner, we get

$$
\begin{aligned}
& \int|I I|^{2} d \mathbf{x}_{k+l_{c}} d \mathbf{x}_{k+l_{c}}^{\prime} \\
= & \int d \mathbf{x}_{k+l_{c}} d \mathbf{x}_{k+l_{c}}^{\prime} \mid \int V_{N}\left(x_{1}-x_{k+l_{c}+1}\right) \\
& \times\left.\left(R^{\left(k+l_{c}\right)} \gamma_{N}^{\left(k+l_{c}+1\right)}\right)\left(t, \mathbf{x}_{k+l_{c}}, x_{k+l_{c}+1} ; \mathbf{x}_{k+l_{c}}^{\prime}, x_{k+l_{c}+1}\right) d x_{k+l_{c}+1}\right|^{2} \\
\leqslant & C N^{3 \beta}\|V\|_{L^{2}}^{2}\left\|S^{\left(k+l_{c}+1\right)} \gamma_{N}^{\left(k+l_{c}+1\right)}\right\|_{L_{T}^{\infty} L_{\mathbf{x}, \mathbf{x}^{\prime}}^{2}}^{2},
\end{aligned}
$$

Accordingly,

$$
\begin{gathered}
\int\left|R^{\left(k+l_{c}\right)} \tilde{B}_{N, 1, k+l_{c}+1} \gamma_{N}^{\left(k+l_{c}+1\right)}(t)\right|^{2} d \mathbf{x}_{k+l_{c}} d \mathbf{x}_{k+l_{c}}^{\prime} \\
\leqslant C N^{5 \beta}\|V\|_{H^{1}}^{2}\left\|S^{\left(k+l_{c}+1\right)} \gamma_{N}^{\left(k+l_{c}+1\right)}\right\|_{L_{T}^{\infty} L_{\mathbf{x}, \mathbf{x}^{\prime}}^{2}}^{2}
\end{gathered}
$$


Thence

$$
\begin{aligned}
& \left\|R^{(k-1)} B_{N, 1, k} I P^{\left(k, l_{c}\right)}\right\|_{L_{T}^{1} L_{\mathbf{x}, \mathbf{x}^{\prime}}^{2}} \\
\leqslant & \sum_{m}\left(C T^{\frac{1}{2}}\right)^{l_{c}}\left\|R^{\left(k+l_{c}\right)} B_{N, \mu_{m}\left(k+l_{c}+1\right), k+l_{c}+1} \gamma_{N}^{\left(k+l_{c}+1\right)}\left(t_{k+l_{c}+1}\right)\right\|_{L_{T}^{1} L^{2} \mathbf{x}, \mathbf{x}^{\prime}} \\
\leqslant & C 4^{l_{c}}\left(C T^{\frac{1}{2}}\right)^{l_{c}} T\left(C N^{\frac{5 \beta}{2}}\|V\|_{H^{1}}\left\|S^{\left(k+l_{c}+1\right)} \gamma_{N}^{\left(k+l_{c}+1\right)}\right\|_{L_{T}^{\infty} L_{\mathbf{x}, \mathbf{x}^{\prime}}^{2}}\right)
\end{aligned}
$$

Put in Condition (2.5), it becomes

$$
\begin{aligned}
\left\|R^{(k-1)} B_{N, 1, k} I P^{\left(k, l_{c}\right)}\right\|_{L_{T}^{1} L_{\mathbf{x}, \mathbf{x}^{\prime}}^{2}} & \leqslant C T\left(C T^{\frac{1}{2}}\right)^{l_{c}} N^{\frac{5 \beta}{2}} C_{0}^{k+l_{c}+1} \\
& =C_{0}^{k}\left[C T\left(C T^{\frac{1}{2}}\right)^{l_{c}} N^{\frac{5 \beta}{2}} C_{0}^{l_{c}+1}\right] .
\end{aligned}
$$

Replace the constants $C$ and $C_{0}$ inside the bracket with some larger constant and group the terms, we have

$$
\left\|R^{(k-1)} B_{N, 1, k} I P^{\left(k, l_{c}\right)}\right\|_{L_{T}^{1} L_{\mathbf{x}, \mathbf{x}^{\prime}}^{2}} \leqslant C_{0}^{k}\left[\left(T^{\frac{1}{2}}\right)^{2+l_{c}} N^{\frac{5 \beta}{2}} C^{l_{c}}\right] .
$$

As in [11, 15], we take the coupling level $l_{c}=\ln N$ to deal with what is inside the bracket

$$
\left\|R^{(k-1)} B_{N, 1, k} I P^{\left(k, l_{c}\right)}\right\|_{L_{T}^{1} L_{\mathbf{x}, \mathbf{x}^{\prime}}^{2}} \leqslant C C_{0}^{k}\left[\left(T^{\frac{1}{2}}\right)^{2+\ln N} N^{\frac{5 \beta}{2}} N^{c}\right] .
$$

Notice that there is no $k$ inside the bracket. Selecting $T$ such that

$$
T \leqslant e^{-(5 \beta+2 C)}
$$

ensures that

$$
\left(T^{\frac{1}{2}}\right)^{\ln N} N^{\frac{5 \beta}{2}} N^{c} \leqslant 1
$$

and thence

$$
\left\|R^{(k-1)} B_{N, 1, k} I P^{\left(k, l_{c}\right)}\right\|_{L_{T}^{1} L_{\mathbf{x}, \mathbf{x}^{\prime}}^{2}} \leqslant C C_{0}^{k} \leqslant C^{k-1}
$$

with a $C$ larger than $C_{0}$ and independent of $k$ and $N$. Whence, we have finished the proof of estimate (2.10).

\section{REFERENCES}

[1] R. Adami, F. Golse, and A. Teta, Rigorous derivation of the cubic NLS in dimension one, J. Stat. Phys. 127 (2007), 1194-1220.

[2] M. H. Anderson, J. R. Ensher, M. R. Matthews, C. E. Wieman, and E. A. Cornell, Observation of Bose-Einstein Condensation in a Dilute Atomic Vapor, Science 269 (1995), 198-201.

[3] W. Beckner, Multilinear Embedding - Convolution Estimates on Smooth Submanifolds, Proc. Amer. Math. Soc. 142 (2014), 1217-1228.

[4] J. Bourgain, Global solutions of nonlinear Schrödinger equations, American Mathematical Society Colloquium Publications, 46. American Mathematical Society, Providence, RI, 1999. viii+182 pp. ISBN: 0-8218-1919-4.

[5] N. Benedikter, G. Oliveira, and B. Schlein, Quantitative Derivation of the Gross-Pitaevskii Equation, to appear in Commun. Pure Appl. Math. (arXiv:1208.0373) 
[6] L. Chen, J. O. Lee and B. Schlein, Rate of Convergence Towards Hartree Dynamics, J. Stat. Phys. 144 (2011), 872-903.

[7] S. L. Cornish, N. R. Claussen, J. L. Roberts, E. A. Cornell, and C. E. Wieman, Stable ${ }^{85}$ Rb BoseEinstein Condensates with Widely Turnable Interactions, Phys. Rev. Lett. 85 (2000), 1795-1798.

[8] T. Chen and N. Pavlović, On the Cauchy Problem for Focusing and Defocusing Gross-Pitaevskii Hierarchies , Discrete Contin. Dyn. Syst. 27 (2010), 715-739.

[9] T. Chen and N. Pavlović, The Quintic NLS as the Mean Field Limit of a Boson Gas with Three-Body Interactions, J. Funct. Anal. 260 (2011), 959-997.

[10] T. Chen and N. Pavlović, A New Proof of Existence of Solutions for Focusing and Defocusing GrossPitaevskii Hierarchies, Proc. Amer. Math. Soc. 141 (2013), 279-293.

[11] T. Chen and N. Pavlović, Derivation of the cubic NLS and Gross-Pitaevskii hierarchy from manybody dynamics in $d=3$ based on spacetime norms, Annales Henri Poincaré 15 (2014), 543-588.

[12] X. Chen, Classical Proofs Of Kato Type Smoothing Estimates for The Schrödinger Equation with Quadratic Potential in $\mathbb{R}^{n+1}$ with Application, Differential and Integral Equations 24 (2011), 209-230.

[13] X. Chen, Second Order Corrections to Mean Field Evolution for Weakly Interacting Bosons in the Case of Three-body Interactions, Arch. Rational Mech. Anal. 203 (2012), 455-497.

[14] X. Chen, Collapsing Estimates and the Rigorous Derivation of the 2d Cubic Nonlinear Schrödinger Equation with Anisotropic Switchable Quadratic Traps, J. Math. Pures Appl. 98 (2012), 450-478.

[15] X. Chen, On the Rigorous Derivation of the 3D Cubic Nonlinear Schrödinger Equation with A Quadratic Trap, Arch. Rational Mech. Anal. 210 (2013), 365-408.

[16] X. Chen and J. Holmer, On the Rigorous Derivation of the 2D Cubic Nonlinear Schrödinger Equation from 3D Quantum Many-Body Dynamics, Arch. Rational Mech. Anal. 210 (2013), 909-954.

[17] P. Clade, C. Ryu, A. Ramanathan, K. Helmerson, and W. D. Phillips, Observation of a 2D Bose Gas: From Thermal to Quasicondensate to Superfluid, Phys. Rev. Lett. 102 (2009) 170401.

[18] K. B. Davis, M. -O. Mewes, M. R. Andrews, N. J. van Druten, D. S. Durfee, D. M. Kurn, and W. Ketterle, Bose-Einstein condensation in a gas of sodium atoms, Phys. Rev. Lett. 75 (1995), 3969-3973.

[19] R. Desbuquois, L. Chomaz, T. Yefsah, J. Léonard, J. Beugnon, C. Weitenberg, J. Dalibard, Superfluid Behaviour of A Two-dimensional Bose Gas, Nature Physics 8 (2012), 645-648.

[20] A. Elgart, L. Erdös, B. Schlein, and H. T. Yau, Gross-Pitaevskii Equation as the Mean Field Limit of Weakly Coupled Bosons, Arch. Rational Mech. Anal. 179 (2006), 265-283.

[21] L. Erdös and H. T. Yau, Derivation of the Non-linear Schrödinger Equation from a Many-body Coulomb System, Adv. Theor. Math. Phys. 5 (2001), 1169-1205.

[22] L. Erdös, B. Schlein, and H. T. Yau, Derivation of the Gross-Pitaevskii Hierarchy for the Dynamics of Bose-Einstein Condensate, Comm. Pure Appl. Math. 59 (2006), 1659-1741.

[23] L. Erdös, B. Schlein, and H. T. Yau, Derivation of the Cubic non-linear Schrödinger Equation from Quantum Dynamics of Many-body Systems, Invent. Math. 167 (2007), 515-614.

[24] L. Erdös, B. Schlein, and H. T. Yau, Rigorous Derivation of the Gross-Pitaevskii Equation with a Large Interaction Potential, J. Amer. Math. Soc. 22 (2009), 1099-1156.

[25] L. Erdös, B. Schlein, and H. T. Yau, Derivation of the Gross-Pitaevskii Equation for the Dynamics of Bose-Einstein Condensate, Annals Math. 172 (2010), 291-370.

[26] J. Fröhlich, A. Knowles, and S. Schwarz, On the Mean-Field Limit of Bosons with Coulomb Two-Body Interaction, Commun. Math. Phys. 288 (2009), 1023-1059.

[27] A. Görlitz, J. M. Vogels, A. E. Leanhardt, C. Raman, T. L. Gustavson, J. R. Abo-Shaeer, A. P. Chikkatur, S. Gupta, S. Inouye, T. Rosenband, and W. Ketterle, Realization of Bose-Einstein Condensates in Lower Dimensions, Phys. Rev. Lett. 87 (2001), 130402.

[28] P. Gressman, V. Sohinger, and G. Staffilani, On the Uniqueness of Solutions to the Periodic 3D GrossPitaevskii Hierarchy, J. Funct. Anal. 266 (2014), 4705-4764.

[29] M. G. Grillakis and D. Margetis, A Priori Estimates for Many-Body Hamiltonian Evolution of Interacting Boson System, J. Hyperb. Diff. Eqs. 5 (2008), 857-883. 
[30] M. G. Grillakis and M. Machedon, Pair excitations and the mean field approximation of interacting Bosons, I, Commun. Math. Phys. 324 (2013), 601-636.

[31] M. G. Grillakis, M. Machedon, and D. Margetis, Second Order Corrections to Mean Field Evolution for Weakly Interacting Bosons. I, Commun. Math. Phys. 294 (2010), 273-301.

[32] M. G. Grillakis, M. Machedon, and D. Margetis, Second Order Corrections to Mean Field Evolution for Weakly Interacting Bosons. II, Adv. Math. 228 (2011) 1788-1815.

[33] Z. Hadzibabic, P. Krüger, M. Cheneau, B. Battelier and J. Dalibard, Berezinskii-Kosterlitz-Thouless crossover in a trapped atomic gas, Nature, 441 (2006), 1118-1121.

[34] M. Keel and T. Tao, Endpoint Strichartz Estimates, Amer. J. Math. 120 (1998), 955-980.

[35] W. Ketterle and N. J. van Druten, Evaporative Cooling of Trapped Atoms, Advances In Atomic, Molecular, and Optical Physics 37 (1996), 181-236.

[36] K. Kirkpatrick, B. Schlein and G. Staffilani, Derivation of the Two Dimensional Nonlinear Schrödinger Equation from Many Body Quantum Dynamics, Amer. J. Math. 133 (2011), 91-130.

[37] S. Klainerman and M. Machedon Space-time estimates for null forms and the local existence theorem, Comm. Pure Appl. Math. 46 (1993), 1221-1268.

[38] S. Klainerman and M. Machedon, On the Uniqueness of Solutions to the Gross-Pitaevskii Hierarchy, Commun. Math. Phys. 279 (2008), 169-185.

[39] A. Knowles and P. Pickl, Mean-Field Dynamics: Singular Potentials and Rate of Convergence, Commum. Math. Phys. 298 (2010), 101-138.

[40] E. H. Lieb, R. Seiringer, J. P. Solovej and J. Yngvason, The Mathematics of the Bose Gas and Its Condensation, Basel, Switzerland: Birkhaüser Verlag, 2005.

[41] A. Michelangeli and B. Schlein, Dynamical Collapse of Boson Stars, Commum. Math. Phys. 311 (2012), 645-687.

[42] P. Pickl, A Simple Derivation of Mean Field Limits for Quantum Systems, Lett. Math. Phys. 97 (2011), 151-164.

[43] I. Rodnianski and B. Schlein, Quantum Fluctuations and Rate of Convergence Towards Mean Field Dynamics, Commun. Math. Phys. 291 (2009), 31-61.

[44] H. Spohn, Kinetic Equations from Hamiltonian Dynamics, Rev. Mod. Phys. 52 (1980), 569-615.

[45] D. M. Stamper-Kurn, M. R. Andrews, A. P. Chikkatur, S. Inouye, H. -J. Miesner, J. Stenger, and W. Ketterle, Optical Confinement of a Bose-Einstein Condensate, Phys. Rev. Lett. 80 (1998), 2027-2030.

[46] S. Stock, Z. Hadzibabic, B. Battelier, M. Cheneau, and J. Dalibard, Observation of Phase Defects in Quasi-Two-Dimensional Bose-Einstein Condensates, Phys. Rev. Lett. 95 (2005), 190403.

Department of Mathematics, Brown University, 151 Thayer Street, Providence, Ri 02912 E-mail address: chenxuwen@math.brown.edu

URL: http://www.math.brown.edu/ ${ }^{\text {chenxuwen/ }}$

Department of Mathematics, Brown University, 151 Thayer Street, Providence, RI 02912

E-mail address: holmer@math.brown.edu

URL: http://www. math. brown.edu/ holmer/ 